\title{
Structural and biochemical analysis of ATPase activity and EsxAB substrate binding of M. tuberculosis EccCb1 enzyme
}

Arkita Bandyopadyay and Ajay K. Saxena\#

Running title: Structure and function of EccCb1 enzyme

Rm-403/440, Structural biology lab, School of Life Sciences, Jawaharlal Nehru University, New Delhi-67, India.

\#Corresponding author: Ajay K. Saxena, Rm-403/440, Structural biology lab, School of Life Sciences, Jawaharlal Nehru University, New Delhi-67, India.

Phone: 26704155, Email:ajaysaxena@ mail.jnu.ac.in 


\section{Abstract}

The EccC enzyme of $M$. tuberculosis ESX-1 system is a promising target for antivirulence drug development. The EccC enzyme comprises two polypeptides (i) EccCa1, a membrane bound enzyme having two ATPase domains D2 \& D3 (ii) cytosolic EccCb, which contains two ATPase domains. In current study, we have analyzed the low-resolution structure of EccCb1, performed ATPase activity and EsxAB substrate binding analysis. The EccCb1 enzyme eluted as oligomer from size exclusion column and small angle X-ray scattering analysis revealed the double hexameric structure in solution. The EccCb1 enzyme showed catalytic efficiency $\left(k_{c a t} / K_{M}\right) \sim 0.020 \pm 0.005 \mu \mathrm{M}^{-1} \mathrm{~min}^{-1}$, however $\sim 3.7$ fold lower than its D2 and $~ 1.7$ fold lower than D3 domains respectively. The D2 and D3 domains exhibited the ATPase activity and mutation of residues involved in $\mathrm{ATP}+\mathrm{Mg}^{2+}$ binding have yielded $56-94 \%$ reduction in catalytic efficiency for both D2 and D3 domains. The EccCb1 binds the EsxAB substrate with $K_{D} \sim 11.4 \pm 3.4 \mathrm{nM}$ via specific groove located at C-terminal region of D3 domain. ATP binding to EccCb1 enhanced the EsxAB substrate binding by $~ 3$ fold, indicating ATPase energy involvement in Esx AB substrate translocation. We modeled the dodecameric EccCb1+EsxAB $+\mathrm{ATP}+\mathrm{Mg}^{2+}$ complex, which showed the binding pockets involved in $\mathrm{ATP}+\mathrm{Mg}^{2+}$ and EsxAB substrate binding. The enzyme dynamics involved in $\mathrm{ATP}+\mathrm{Mg}^{2+}$ and EsxAB substrate recognition were identified and showed the enhanced stability of EccCb1 enzyme as a result of ligand binding. Overall, our structural and biochemical analysis showed the low-resolution structure and mechanism involved in ATPase activity and EsxAB substrate binding and dynamics involved in EsxAB substrate and $\mathrm{ATP}+\mathrm{Mg}^{2+}$ recognition. Overall, our structural and biochemical data on EccCb1 will 
contribute significantly in development of antivirulence inhibitors, which will prevent virulence factor secretion by $M$. tuberculosis ESX-1 system.

Keywords: M. tuberculosis ESX-1, EccCb1, EsxAB, ATPase activity, Binding analysis, Small angle X-ray scattering, Modeling and dynamics simulation 


\section{Introduction}

M. tuberculosis ESX-1 system (early secretory antigenic target 6 system-1) is involved in virulence factor secretion into host cell, which help mycobacterial survival in the macrophage. Loss of ESX-1 system in mycobacteria leads to genetic difference between virulent and live attenuated strains [1]. M. tuberculosis type VII secretion system (T7SS, 1.5 MDa protein complex) contains five different ESX 1 to 5 systems and involved in various physiological functions, including virulence factor secretion [2]. The function of ESX-2 and ESX-4 secretion systems are currently unknown. Recently, structure of ESX-3 secretion system was determined [3-4], which identified an additional domain in EccC enzyme, following by transmembrane helices, a stalk domain and fourth ATPase (DUF) domain. The structure of ESX-5 secretion system was also determined at $13 \AA$ resolution using electron microscopy technique [5]. It indicates the architecture of type VII secretion system is quite different than other ESX secretion systems.

The M. tuberculosis EccC enzyme belongs to ESX-1 secretion system and contains (i) a cytosolic EccCb1 enzyme (Mw $64.5 \mathrm{kDa}, 591$ residues) and (ii) a membrane anchored EccCa1 enzyme and belong to FTSK/SpoIIIE family of mechanoenzymes involved in DNA translocation during bacterial cell division and activator of XerCD site specific recombination $[6,7]$. The EccCa1 enzyme contains two transmembrane helices, two cytosolic ATPase domain and forms complex with EccCb1enzyme and export EsxAB substrate out of the cell $[2,6]$. The EccCb1 enzyme contains two P-loop NTPase domains, as observed in ACSE (Additional Strand Conserved Glutamate) family of proteins e. g. Ftsk, TrwB and TrwK [8]. The ASCE family of enzymes harbor the residues, which enters into 
active site of neighboring domain upon multimerization [9]. The M. tuberculosis EccC enzyme consists of two polypeptides, while single polypeptide is observed for T. curvata EccC enzyme [10] and G. thermodentrificans EssC enzyme [11]. In T. curvata EccC enzyme, linker between ATPase1 and ATPase2 domains was found quite similar to the linker between ATPase2 and ATPase3 domains and play key role in regulation of ATPase1 domain activity.

M. tuberculosis EsxAB substrate belongs to WxG superfamily of proteins [12-13]. The WxG family of proteins form characteristic homo or heterodimer, in which one monomer contains the $\mathrm{WxG}$ motif and another monomer contains the YxxxD/E motif in its export arm. The export arm of EsxAB substrate binds to EccCb1 enzyme for its secretion out of the cell [14]. The ESX-1 substrates secrete in codependent manner, as M. tuberculosis strains lacking the EsxAB substrate were unable to secrete other virulence factors out of the cell [15].

Recently, crystal structure of C-terminal D3 domain of M. tuberculosis EccCb1 enzyme in complex with peptide from EsxB substrate was determined at $2.2-\AA$ resolution [16]. The structure analysis showed the nature and location of substrate binding pocket of EccCb1 enzyme. In another study, crystal structure of ATPase domain of EssC enzyme from S. aureus USA300_FPR3757 was determined at 1.7-A resolution [17], which also elucidated the structure of substrate binding pocket of the enzyme.

To understand the structure and mechanism of EccCb1 enzyme, we have determined the low-resolution structure of EccCb1 using small angle X-ray scattering technique. The ATPase activity of full length Rv3871 and its D2 and D3 domains were determined and 
performed comparative analysis. The EsxAB substrate binding to EccCb1was determined and enzyme dynamics involved in $\mathrm{ATP}+\mathrm{Mg}^{2+}$ and Esx $\mathrm{AB}$ substrate recognition were determined using dynamics simulation technique. Our structural and biochemical data on EccCb1 can be targeted for anti-virulence inhibitors development, which will prevent virulence factor secretion by M. tuberculosis ESX-1 system.

\section{Results}

\section{Full length EccCb1 forms dodecamer in solution and exhibited specific ATPase activity}

The schematic view of full-length EccCb1 enzyme (65 kDa, 591 residues) is shown in Fig. 1A. The EccCb1 consists of two polypeptides, D2 domain (35-316 residues) and D3 domain (349-580 residues), which belong to P- type NTPase family. The ATP binding motif of D2 domain $\left({ }^{84}\right.$ GAPQTGKS ${ }^{91}$, cyan $)$ and D3 domain $\left({ }^{376}\right.$ GAAKSGKT $^{383}$, brown $)$ are shown in

Fig. 1A. Full length EccCb1 were purified, however eluted as oligomer on Superdex $200^{\mathrm{TM}}$ (16/60) column, identified based on molecular mass standard (Fig. 1B, inset). The eluted protein from column showed purity more than $98 \%$ and single band on SDS-PAGE analysis

(Fig. 1B, inset). Small angle X-ray scattering analysis on Rv3871 showed double hexameric structure $(\mathrm{Mw} \sim 780 \mathrm{kDa})$ in solution.

$\left[\gamma_{-}{ }^{32} \mathrm{P}\right]$ radioactivity assay was performed to analyze the ATPase activity of EccCb1 enzyme (Fig. 1C). In radioactivity assay, release of free Phosphate was monitored gradually after every $15 \mathrm{~min}$ and showed maximum ATP hydrolysis (20\%) at $60 \mathrm{~min}$ (Fig. 1C). Three measurements were taken for each reading and reaction buffer containing no EccCb1 taken as negative control. Calorimetric assay showed the ATPase activity of purified EccCb1 and following parameters e. g. $V_{\max } \sim 9.28 \mu \mathrm{M} \min ^{-1} \mathrm{mg}^{-1}, K_{m} \sim 0.02 \pm 0.01 \mathrm{mM}$ and $k_{\text {cat }} / K_{m} \sim$ 
$0.020 \pm 0.005 \mu \mathrm{M}^{-1} \mathrm{~min}^{-1}$ were obtained using Michaelis-Menten plot (Fig. 1D) and Graph Pad Prism 6.0 software [18].

To examine the effect of various parameters e. g. oligomerization, EDTA, $\mathrm{MgCl}_{2}$ on EccCb1 ATPase activity, $\left[\gamma^{32} \mathrm{P}\right]$ radioactivity assay was performed using these compounds. In presence of $500 \mathrm{mM} \mathrm{NaCl}$ in reaction buffer, the EccCb1 showed 50\% decrease in ATP hydrolysis. In presence of $10 \mathrm{mM}$ EDTA, the EccCb1 showed no ATP hydrolysis indicating absolute requirement of $\mathrm{Mg}^{2+}$ for enzyme activity. $\mathrm{Mg}^{2+}$ binds to $\beta$ - and $\gamma-\mathrm{PO}_{4}$ of $\mathrm{ATP}$ and neutralize the negative charge on $\mathrm{PO}_{4}$ group and promotes the nucleophilic attack by activated water for ATP hydrolysis. In presence of $1 \mathrm{mM} \mathrm{MgCl}$, EccCb1 showed only $2 \%$ ATP hydrolysis compared to wild type enzyme (Fig. 1E).

To analyze the effect of various ions on percentage of ATP hydrolysis, $\left[\gamma^{32} \mathrm{P}\right]$ radioactivity assay was performed and (\%) of ATP hydrolysis was calculated. $25 \%$ ATP hydrolysis was observed with $5 \mathrm{mM} \mathrm{Mn}^{2+} 8 \%$ with $5 \mathrm{mM} \mathrm{Ca}^{2+}$ and $20 \%$ with $5 \mathrm{mM} \mathrm{Mg}^{2+}$ (Fig. 1F). These data showed that $\mathrm{Mn}^{2+}$ ion (radii 0.65 $)$ ) fit better into ATPase pocket than $\mathrm{Mg}^{2+}$ ion (ionic radii $\sim 0.46 \AA$ ), which resulted in higher ATPase activity of EccCb1 enzyme. However, $\mathrm{Ca}^{2+}$ (ionic radii $\sim 1.11 \AA$ ) is higher than $\mathrm{Mg}^{2+}$ (radii $\sim 0.46 \AA$ ) and may not be fitting optimally into ATPase pocket, which result in lower ATPase activity of EccCb1 enzyme.

\section{Small angle X-ray scattering analysis yielded the double hexametric ring structure of}

\section{EccCb1 in solution}

To determine the low-resolution structure of EccCb1, small angle X-ray scattering experiment was performed. The SAXS profile obtained in double logarithmic mode (Fig. 
2A) supported a monodisperse profile of EccCb1 in solution, devoid of any aggregation or interparticulate effect. Considering the monodisperse globular shape of EccCb1, linearity in the Guinier region was observed (Fig 2A, inset). Globular scattering profile of EccCb1 in solution was confirmed by peak close to 1.75 in normalized Kratky plot (Fig. 2B). The Guinier and Kratky plots analysis yielded the radius of gyration $\left(\mathrm{R}_{\mathrm{g}}\right) \sim 6.87 \mathrm{~nm}$ for EccCb1. Presuming the rod-shape structure of EccCb1, the Guinier analysis showed the crosssectional radius, $\mathrm{R}_{\mathrm{c}} \sim 6.77 \mathrm{~nm}$. Considering wider q range of data, $\mathrm{P}(\mathrm{r})$ analysis on $\mathrm{EccCb} 1$ yielded the $\mathrm{D}_{\max } \sim 20.14 \mathrm{~nm}$ and $\mathrm{R}_{\mathrm{g}} \sim 6.86 \mathrm{~nm}$ (Fig. 2C). Peak-shoulder of the $\mathrm{P}(\mathrm{r})$ curve showed single lobe of scattering (Fig. 2C). Using dummy residue models and shape restoration using SAXS data-based constraints showed an envelope compatible to double hexameric ring structure of EccCb1 (Fig. 2D). The NSD value $\sim 0.883 \pm 0.132$ was observed for selected model with resolution $\sim 9.7 \pm 0.7 \mathrm{~nm}$. The theoretical $R_{g}$ and $D_{\max }$ were quite compatible to the experimental $R_{g}$ and $D_{\max }$ values.

The $a b$ initio envelope of EccCb1 was obtained from SAXS data. It indicates the presence of double hexameric ring structure of EccCb1 in solution. We modeled the double hexameric ring structure of EccCb1, which fitted very well into acquired SAXS envelope (Fig. 2D-G). The CRYSOL program [19] was used to compare theoretical SAXS profiles from model vs experimental SAXS data, which yielded quite good fitting $\left(\chi^{2} \sim 0.85\right)$ (Fig. 2H). The details of SAXS data collection and various parameters used in structure solution are given in Table 3 .

\section{D2 domain forms dimer in solution and exhibited ATPase activity}

The web logo diagram [20] of sequence alignment of ATPase motif of D2 domain with 80 
heterologous EccC sequences is shown in Fig. 3A. These data showed that ${ }^{90} \mathrm{GKS}{ }^{92}$ sequence of ATPase motif is $100 \%$ conserved in all $80 \mathrm{EccC}$ sequences. The D2 domain (35-316 residues, $31 \mathrm{kD})$ was purified and eluted as dimer from Superdex $200^{\mathrm{TM}}(16 / 60)$ column, as identified based on molecular mass standard (Fig. 3B, inset). The D2 domain showed purity more than $98 \%$ and appeared as single band on SDS-PAGE analysis (Fig. 3B, inset). Four site directed mutants of D2 domain were prepared and all mutants eluted as dimer like wild type D2 domain (Fig. 3B).

ATPase activity of D2 domain was determined using calorimetric assay and following parameters $\left(K_{m} \sim 0.03 \pm 005 \mathrm{mM}, V_{\max } \sim 63.79 \mu \mathrm{M} / \mathrm{min}\right.$ and catalytic efficiency $\left.\left(K_{\text {cat }} / K_{m}\right) \sim 0.070 \pm 0.020 \mu \mathrm{M}^{-1} \mathrm{~min}^{-1}\right)$ were obtained using Michaelis-Menten plot and nonlinear regression analysis using Prism 6.0.2 GraphPad Software (Fig. 3D). We modeled the D2 domain $+\mathrm{ATP}+\mathrm{Mg}^{2+}$ complex based on PDB-4N1A [10] structure as input and identified key residues involved in ATP and $\mathrm{Mg}^{2+}$ binding using LigPlot program [21] (Fig. 3C). The Gln87, Lys90, Ser91 and Thr92 residues of D2 domain form hydrogen bonds with $\gamma$-PO4 group of ATP and Thr92 and Ile285 residues form hydrogen bonds with adenine ring of ATP.

To understand the roles in ATPase activity, we generated the Q87A, K90A, S91A and T92A D2 mutants and analyzed the ATPase activity (Fig. 3D). Table 1 showed the kinetic parameters observed for four D2 mutants. The Q87A mutation leads to ( $~ 55.9 \%$ decrease in catalytic efficiency and $\sim 1.5$-fold decrease in $\mathrm{V}_{\max }$ ). The K90A mutation leads to $\left(\sim 93.9 \%\right.$ decrease in catalytic efficiency and $\sim 2.6$-fold decrease in $\left.\mathrm{V}_{\max }\right)$. The $\mathrm{S} 91 \mathrm{~A}$ mutation leads to $\left(\sim 42.2 \%\right.$ decrease in catalytic efficiency and $\sim 1.4$-fold decrease in $\left.\mathrm{V}_{\max }\right)$. The T92A mutation leads to ( 82.3\% decrease in catalytic efficiency and quite similar 
$\mathrm{V}_{\max }$ ) compared to wild type D2 domain. These data showed that K90A mutation critically affect the ATPase activity and leads to almost complete loss of D2 domain activity. The wild type and four D2 mutants were expressed and purified under similar conditions and showed no abnormal elution behavior on size exclusion column (Fig. 3B). Differences in ATPase activity of four D2 mutants were not due to defect in folding or aggregation of proteins.

\section{D3 domain forms dimer in solution and exhibited ATPase activity}

Web logo diagram of sequence alignment of ATPase motif of D3 domain with 80 heterologous EccC sequences is shown in Fig. 4A. These data showed that Gly382 and Lys383 residues of D3 domain were $86 \%$ and $84 \%$ conserved in all EccC sequences. The D3 domain eluted as dimer $(\mathrm{Mw} \sim 50 \mathrm{kDa})$ from Superdex $200^{\mathrm{TM}}(16 / 60)$ column, as identified based on molecular mass standard (Fig. 4B, inset). Purified D3 domain showed purity more than $98 \%$ and appeared as single band on SDS-PAGE analysis (Fig. 4B, inset).

The ATPase activity of D3 domain was determined using calorimetric assay and following parameters $\left(K_{m} \sim 0.06 \pm 0.03 \mathrm{mM}\right.$, catalytic efficiency $\left(k_{c a t} / K_{m)} \sim\right.$ $0.030 \pm 0.008 \mu \mathrm{M}^{-1} \min ^{-1}$ and $V_{\max } \sim 52.89 \mu \mathrm{M} / \mathrm{min}$ ) were obtained (Fig. 4D). Residues involved in ATP and $\mathrm{Mg}^{2+}$ binding were identified from M. tuberculosis ATPase3 domain+ peptide $+\mathrm{ATP}+\mathrm{Mg}^{2+}$ crystal structure (PDB-6J19). LigPlot analysis (Fig. 4C) showed that K382, T383, T384 residues of D3 domain bind to $\mathrm{PO}_{4}$ moiety and A574 and Y576 residues to Adenine ring of ATP.

To understand the roles of these residues in ATPase activity, K382A, T383A, T384A, A574G and Y576A mutants were generated using site directed mutagenesis (Fig. 
4C) and ATPase assay was performed on all 5 mutants. Table 1 showed the kinetic parameters obtained for 5 D3 mutants. These data showed that K382A mutation leads to ( $93.4 \%$ decrease in catalytic efficiency, $80 \%$ decrease in $\mathrm{V}_{\max }$ ). The T383A mutation leads to $\left(\sim 89.7 \%\right.$ decrease in catalytic efficiency, $\sim 50 \%$ decrease in $\left.\mathrm{V}_{\max }\right)$. The T384A mutation leads to $\left(\sim 87.3 \%\right.$ decrease in catalytic efficiency and $\sim 22 \%$ decrease in $\left.\mathrm{V}_{\text {max. }}\right)$. The T574A mutation leads to $\left(\sim 73.5 \%\right.$ decrease in catalytic efficiency and $\sim 12 \%$ decrease in $\left.\mathrm{V}_{\max }\right)$ and Y576A mutation leads to $(\sim 75.2 \%$ decrease in catalytic efficiency and $\sim 15 \%$ decrease in $\mathrm{V}_{\max }$ ), compared to wild type D3 domain. These data showed that K382A mutation is the most critical and leads to $93.4 \%$ decrease in catalytic efficiency of the enzyme. The wild type and five D3 mutants were expressed and purified under similar condition and showed no abnormal migration on size exclusion column (Fig. 4B). In all D3 mutants, difference in ATPase activities were not due to defect in folding or aggregation of proteins.

We have compared the kinetic parameters of D2 and D3 domains with full length EccCb1enzyme (Table 1). These data showed that D2 domain exhibited $\sim 6.9$-fold higher $\mathrm{V}_{\max }$ and $\sim 3.4$ fold higher catalytic efficiency. The D3 domain exhibited $\sim 5.6$ fold higher $\mathrm{V}_{\max }$ and $\sim 1.7$ fold higher catalytic efficiency compared to wild type EccCb1 enzyme.

\section{Modeling of EccCb1+ATP+Mg ${ }^{2+}+$ EsxAB complex and EsxAB substrate binding analysis}

The EccCb1+ATP+Mg ${ }^{2+}$ model was obtained using PDB-4N1A [10] and PDB-6J19 [16] structures as input templates (as described in Materials and Methods section). The topology diagram of EccCb1 (Fig. 5A) showed the arrangement of $\alpha$-helices (red) and $\beta$-sheets (pink) in EccCb1enzyme and numbered accordingly. The EccCb1+ ATP $+\mathrm{Mg}^{2+}+\mathrm{EsxAB}$ model 
contains total 28-580 residues, in which D2 domain contains (35-316 residues) and D3 domain contains (349-580 residues) and connected by linker of 317-348 residues (Fig. 5B). The D2 and D3 domains contain core 8 stranded central $\beta$-sheets, decorated by $\alpha$-helices on either side of $\beta$-sheets. The EsxAB substrate binds to specialized groove at $\mathrm{C}$-terminal region of D3 domain (Fig. 5B). PDBsum server [22] analysis showed the single hydrogen bond and multiple van der waals interactions involved in EsxB substrate binding (Fig. 5C). Binding analysis between EccCb1 and EsxAB substrate was performed using bilayer interferometry, which yielded the $K_{D} \sim 34.4 \mathrm{nM}$ (Fig. 5D). In presence of $0.02 \mathrm{mM}$ ATP in buffer, the $K_{D} \sim 11.4 \mathrm{nM}$ was observed (Fig. 5E). These data showed that EccCb1 binds strongly to EsxAB substrate, and its affinity enhanced $~ 3$-fold towards substrate in presence of ATP, indicating ATPase energy involvement in substrate translocation.

\section{EccCb1 sequence alignment and comparative structure analysis}

Sequence alignment of EccCb1 (1-591 residues) with T. curvata EccC enzyme (285-861 residues, PDB-4N1A) showed $39.8 \%$ sequence identity and $20.3 \%$ identity with and $G$. thermodentroficans EccC enzyme (1-419 residues, PDB -4LYA). The P-loop residues of D2 and D3 domains (shown as \#) were found quite conserved to T. curvata EccC and $G$. thermodentroficans EccC enzyme sequences (Fig. 6A) and with 80 heterologous EccC sequences (Fig. S3). Superposition of EccCb1 structure on T. curvata EccC structure (PDB4N1A) has yielded RMSD $0.78 \AA$ for $461 \mathrm{C} \alpha$ atoms and RMSD $\sim 7.1 \AA$ for $428 \mathrm{C} \alpha$ atoms with G. thermodentroficans EccC (PDB-4LYA) enzyme. The core $\alpha$-helices and $\beta$-sheets of EccCb1 superposed very well with both enzymes, except minor differences in loop regions (Fig. 6B). In EccCb1 enzyme, D2 domain (28-316 residues) showed xxx identity 
with D3 domain (349-580) and superposition both domains yielded RMSD $5.7 \AA$ over 146 C $\alpha$ atoms (Fig. 6C).

The double hexameric EccCb1+EsxAB+ATP+Mg ${ }^{2+}$ complex model was obtained as shown in (Materials and methods section) (Fig. 6D). The double hexameric model showed the following dimensions, [21 (inner) x $94 \AA$ (outer)] diameter for D2 ring and [21 (inner) x $102 \AA$ (outer)] diameter for D3 ring and $80 \AA$ A height of the model (Fig. 6D).

\section{Dynamics simulation analysis revealed the EccCb1 dynamics involved in EsxAB} substrate and $\mathrm{ATP}+\mathrm{Mg}^{2+}$ recognition

To study the EccCb1 dynamics involved in $\mathrm{ATP}+\mathrm{Mg}^{2+}$ and EsxAB substrate recognition, 1 ns dynamics simulation was performed on EccCb1 dodecamer in three environments, (i) as apo enzyme (ii) complex with $\mathrm{ATP}+\mathrm{Mg}^{2+}$ and (iii) complex with $\mathrm{ATP}+\mathrm{Mg}^{2+}+\mathrm{EsxAB}$ substrate (Table 4). The simulated EccCb1 models were superposed on starting structures and conformational changes observed during simulation were analyzed (Fig. 7A-C).

The residual flexibility of apo, $\mathrm{ATP}+\mathrm{Mg}^{2+}$ bound and $\mathrm{ATP}+\mathrm{Mg}^{2+}+\mathrm{EsxAB}$ bound EccCb1 dodecamer are shown in Fig. 7D. For apo EccCb1 dodecamer (cyan), the RMSD of backbone $\mathrm{C} \alpha$ atoms go from 0 to $5 \mathrm{~nm}$ in $200 \mathrm{ps}$ and remained stable to $8 \mathrm{~nm}$ throughout 1 ns simulation and similar results were obtained for $\mathrm{ATP}+\mathrm{Mg}^{2+}$ bound $\mathrm{EccCb} 1$ dodecamer (red). For EsxAB $+\mathrm{ATP}+\mathrm{Mg}^{2+}$ bound complex (blue), the RMSD of backbone C $\alpha$ atoms start from $19 \mathrm{~nm}$ and remained stable throughout $1 \mathrm{~ns}$ simulation. These data showed that backbone $\mathrm{C} \alpha$ fluctuations were maximum for wild type and $\mathrm{ATP}+\mathrm{Mg}^{2+}$ bound $\mathrm{EccCb} 1$ dodecamer during 1 ns simulation. However, backbone $\mathrm{C} \alpha$ fluctuations reduced significantly upon EsxAB substrate binding to EccCb1 ATP $+\mathrm{Mg}^{2+}$ dodecamer. 
The compactness $\left(\mathrm{R}_{\mathrm{g}}\right)$ and associated stability of apo, $\mathrm{ATP}+\mathrm{Mg}^{2+}$ and $\mathrm{ATP}+\mathrm{Mg}^{2+}+\mathrm{Esx} \mathrm{AB}$ bound EccCb1 dodecamer are shown in Fig. 7E. The radius of gyration of EccCb1 models remain constant during $1 \mathrm{~ns}$ simulation. Following radius of gyration $6.2 \mathrm{~nm}$ for wild type, $~ 7.0 \mathrm{~nm}$ for $\mathrm{ATP}+\mathrm{Mg}^{2+}$ bound and $\sim 13 \mathrm{~nm}$ for EsxAB $+\mathrm{ATP}+\mathrm{Mg}^{2+}$ bound EccCb1 dodecamer were observed during dynamics simulation. These data showed that compactness (stability) of EccCb1 dodecamer increased as a result of $\mathrm{ATP}+\mathrm{Mg}^{2+}$ and EsxAB+ATP $+\mathrm{Mg}^{2+}$ binding to enzyme.

The RMSF of the backbone $\mathrm{C} \alpha$ atoms were calculated for EccCb1 models and examined the atomic fluctuations occurred during 1 ns simulation (Fig. 7F). Following RMSF values were obtained e. g. 0.2-0.4 nm for apo (cyan), 0.2-0.4 nm for ATP $+\mathrm{Mg}^{2+}$ bound (red) and 0.2-0.8 $\mathrm{nm}$ for EsxAB+ATP+ $\mathrm{Mg}^{2+}$ bound (blue) EccCb1 dodecamer.

Superposition of simulated EccCb1 dodecamer on starting EccCb1 dodecamer (Fig. 7A) have yielded following (i) RMSD $1.37 \AA$ (6636 C $\alpha$ atoms) for wild type (ii) $\sim 1.65 \AA$ (6636 C $\alpha$ atoms) for ATP+Mg ${ }^{2+}$ bound (Fig. 7B) and (iii) $0.46 \AA$ for 6636 enzyme $\mathrm{C} \alpha$ atoms and $\sim 2.7 \AA$ for 2184 ligand $\mathrm{C} \alpha$ atoms $\mathrm{f}$ in case of EsxAB+ATP+Mg ${ }^{2+}$ bound EccCb1 dodecamer (Fig. 7C). The secondary structures of EccCb1 dodecamer superposed well with starting structure in all models and large movement occurred in the loop region of EccCb1 dodecamer. Major changes were observed in orientations of EsxAB heterodimer during dynamics simulation.

\section{Discussion}

In current study, we have analyzed the SAXS structure, ATPase activity and EsxAB substrate binding of EccCb1 enzyme. Key residues of D2 and D3 domains of EccCb1 
enzyme involved in ATPase activity were identified and characterized using wild type and mutant enzymes. De novo low-resolution envelope of EccCb1 was determined using small angle X-ray scattering technique, which fitted very well with modeled double EccCb1+ATP $+\mathrm{Mg}^{2+}$ hexameric structure. The EsxAB substrate binds to EccCb1 dodecamer with $\mathrm{nM}$ affinity and a specific pocket at C-terminal region of D3 domain was involved in binding. The EccCb1 dynamics involved in $\mathrm{ATP}+\mathrm{Mg}^{2+}$ and Esx AB substrate recognition was analyzed using dynamics simulation technique. Our structural and biochemical analysis on EccCb1will contribute significantly in development of antivirulence inhibitors, which will prevent virulence factor secretion by ESX-1 system.

The AAA+ ATPases enzymes usually oligomerize to remain in active form. The AAA+/RecA ATPases usually form active hexamer in solution, a common characteristic of this family of enzymes. The G. thermodenitrificans EssC oligomerizes in absence of EsxB substrate. However, T. curvata EccC enzyme multimerizes upon binding to EsxB substrate. In our case, EccCb1 eluted as oligomer from size exclusion column and appeared as double hexameric envelope in solution using small angle X-ray scattering data. The EccCb1 does not require Esx $\mathrm{AB}$ substrate for its multimerization, as observed for T. curvata EccC enzyme.

The full length EccCb1 at its D2 and D3 domains have exhibited ATPase activity. In D2 and D3 domains, residues involved in ATPase activity were identified and characterized using wild type and mutantD2 and D3 domains. The K90A mutation in walker A motif of D2 domain and K382A mutation in walker A motif of D3 domain leads to 94\% decrease in enzyme activity. In T. curvata EccC enzyme, T92A mutation in walker A motif of D2 domain and T383A mutation in D3 domain also lead to 90\% decrease in enzyme activity, 
The D2 and D3 domains showed higher ATPase activity than full length of EccCb1 enzyme. Similar results were obtained for G. thermodenitrificans EssC enzyme, which hydrolyze ATP at much lower rate, compared to its D2 and D3 domains. In T. curvata EccC enzyme, both D2 and D3 domains bind to ATP+Mg ${ }^{2+}$ and showed no ATPase activity. In G. dentrificans EssC enzyme, D3 domain does not bind to ATP. In Staphylococcus EssC enzyme, the D3 domain lacks the ATP binding motif, as its P-loop is converted into helix and occluded the ATP binding.

The EsxAB binds to EccCb1at specialized groove at C-terminal region of D3 domain, distant from ATPase pocket. ATP binding to EccCb1 enhanced its affinity towards EsxAB substrate, indicating ATPase energy involvement in EsxAB substrate translocation. In T. curvata EccC enzyme, the EsxB substrate also binds to D3 domain, which leads to enzyme multimerization. In VirD4 ATPase enzyme [23], the C-terminal region of substrate binds to enzyme before its secretion. In G. dentrificans EssC enzyme, the EsxB substrate binds to D3 domain, which induces its multimerization. In T. curvata EccC enzyme, ATP binding does not show any effect on EsxB substrate binding.

Low resolution envelope of EccCb1 was obtained using SAXS data, which fitted well with double hexameric EccCb1structure (Fig. 5D). The double hexameric structures were also obtained in other AAA+ATPases enzymes e. g. PatFtsk docecamer [24], archael MCM dodecamers [25] and SV40-L tag protein [26]. It appears that AAA+ATPases enzymes have propensity to form higher ordered hexamer upon oligomerization and may form a substrate transport channel powered by its ATPase energy.

In EccCb1+Esx AB $+\mathrm{ATP}+\mathrm{Mg}^{2+}$ dodecamer, twelve D2 and D3 domains contain specific pockets and bind to $\mathrm{ATP}+\mathrm{Mg}^{2+}$ binding, however specific groove involved in 
EsxAB substrate binding was observed in D3 domain only. Comparative structure analysis of EccCb1+ATP+Mg ${ }^{2+}$ monomer with two homologous T. curvata EccC and G. dentrifican EssC enzymes showed the overall conserved structure between enzymes and highly conserved P-loop involved in ATPase activity.

Dynamics simulation analysis on apo, $\mathrm{ATP}+\mathrm{Mg}^{2+}$ bound and EsxAB $+\mathrm{ATP}+\mathrm{Mg}^{2+}$ bound EccCb1 dodecamer showed the enzyme dynamics involved in $\mathrm{ATP}+\mathrm{Mg}^{2+}$ and EsxAB substrate recognition. The $\mathrm{ATP}+\mathrm{Mg}^{2+}$ binding enhanced the overall stability of EccCb1 dodecamer, as ATPase site residues showed lower RMSF values compared to apo enzyme. The EsxAB substrate binding further stabilized the substrate-binding groove ofD3 domain, as low RMSF values were observed for substrate binding residues. Our in-silico studies have rationalized the biochemical data observed for EccCb1 ATPase activity and EsxAB substrate binding. In summary, our structural and biochemical data on EccCb1 will provide new avenues for the development of antivirulence inhibitors to block virulence factor -1 system.

\section{Materials and Methods}

\section{Expression and purification}

Preparation of full length EccCbl enzyme

Full-length EccCb1 gene (encoding Met1-Glu591 residues) was amplified using polymerase chain reaction using $M$. tuberculosis $H 37 R v$ genomic DNA. The amplified gene was cloned into $p M A L-p 2 X$ vector using BamH1 and HindIII restriction sites. The resulting $p M A L$ $E c c C b 1$ plasmid contains the genes for maltose binding protein and factor Xa enzyme at Nterminus of EccCb1 gene. The EccCb1 plasmid was verified by gene sequencing and 
restriction-digestion analysis. The $p M A L-E c c C b 1$ plasmid was transformed in E. coli. ER2508 cells and single colony was grown in terrific broth media (Ampicillin $~ 100 \mu \mathrm{g} / \mathrm{ml}$ ) at $37^{\circ} \mathrm{C}$, till $\mathrm{OD}_{600} \sim 0.6-0.7$. The cell culture was induced with $0.5 \mathrm{mM}$ IPTG at $15^{\circ} \mathrm{C}$ and grew the cells for another $18 \mathrm{hr}$. The MBP-EccCb1 fusion protein was overexpressed in soluble fraction of cell.

The cells were centrifuged at $8000 \mathrm{rpm}$ for $3 \mathrm{~min}$ at $4{ }^{0} \mathrm{C}$ and suspended in lysis buffer-A (25mM Tris-HCl pH 7.5, 500 mM NaCl, 1 mM EDTA, 15\%(v/v) Glycerol, 10mM Arginine, $4 \mathrm{mM} \beta$-mercaptoethanol, 1mM Phenylmethylsulfonyl fluoride, $2 \mathrm{mM}$ Benzamidine hexachloride, $0.3 \mathrm{mM}$ ATP, $0.2 \mathrm{mg} / \mathrm{ml}$ Lysozyme). The cell lysate was homogenized, sonicated for $4 \mathrm{~min}$ and subjected to $25,000 \mathrm{x}$ g centrifugation for $30 \mathrm{~min}$ to collect the supernatant. The supernatant was mixed with amylose resin (from NEB), preequilibrated with buffer-B (Buffer-A with no lysozyme) and loaded in empty column. The column was washed with buffer-B $+0.3 \mathrm{mM}$ Maltose. The MBP-EccCb1 fusion protein was eluted in buffer-B $+5 \mathrm{mM}$ Maltose.

For MBP tag cleavage, MBP-EccCb1 fusion protein was mixed with Factor Xa enzyme at [1000:1 (w/w) ratio] and dialyzed in buffer $(20 \mathrm{mM}$ Tris-HCl pH 8.0, $100 \mathrm{mM}$ $\mathrm{NaCl}, 2 \mathrm{mM} \mathrm{CaCl}$ ) for $12 \mathrm{hr}$ at $4^{0} \mathrm{C}$. The cleavage reaction was stopped with $2 \mathrm{mM}$ PMSF and reaction was analyzed on $8 \%$ SDS-PAGE. The reaction mixture was loaded on amylose column and cleaved EccCb1 was eluted from column. The cleaved EccCb1 was loaded on Superdex $200^{\mathrm{TM}}(16 / 60)$ column (from GE Healthcare Ltd), preequilibrated with buffer (25 $\mathrm{mM}$ Tris- $\mathrm{HCl} \mathrm{pH}$ 7.5, $300 \mathrm{mM} \mathrm{NaCl}, 15 \%$ (v/v) Glycerol, $10 \mathrm{mM}$ Arginine, $10 \mathrm{mM} \mathrm{MgCl}$, $0.3 \mathrm{mM}$ ATP, $4 \mathrm{mM} \beta$-mercaptoethanol). The EccCb1 fractions were pooled and concentrated using $30 \mathrm{kDa}$ cutoff ultracentrifugal device (Millipore). The protein 
concentration was determined using Bradford assay and purity was analyzed on 8\% SDS-

PAGE.

\section{Preparation of wild type and mutant D2 domains}

The D2 domain gene (encoding 35-316 residues) was amplified using polymerase chain reaction using $M$. tuberculosis $H 37 R v$ genomic DNA. The amplified D2 domain gene was cloned into pET23a (+) expression vector using Nde1 and XhoI restriction sites. The pET23a-D2 domain gene plasmid was verified by gene sequencing and restriction-digestion analysis. The D2 plasmid was transformed in E. coli. BL21(DE3)Plys cells (Ampicillin $100 \mu \mathrm{g} / \mathrm{ml}$ ) and single colony was grown in terrific broth media, till $\mathrm{OD}_{600} \sim 0.6-0.7$. The culture was induced with $1 \mathrm{mM}$ IPTG at $25^{\circ} \mathrm{C}$ and grown for another $14 \mathrm{hr}$. The cells were harvested at $8000 \mathrm{rpm}$ at $4{ }^{0} \mathrm{C}$ and suspended in lysis buffer-A $(50 \mathrm{mM}$ Tris- $\mathrm{HCl} \mathrm{pH} 7.5$, 300mM NaCl, 15\% Glycerol, 4 mM $\beta$-mercaptoethanol, 1 mM Phenylmethylsulfonyl fluoride, 2mM Benzamidinehexachloride, 10mM $\mathrm{MgCl}_{2}$, $0.3 \mathrm{mM}$ ATP, $0.2 \mathrm{mg} / \mathrm{ml}$ Lysozyme and 10mM Arginine). The cell lysate was homogenized, sonicated and centrifuged at 25,000 $\mathrm{x} g$ to collect the supernatant.

The supernatant was loaded on Ni-NTA column (Sigma), preequilibrated with buffer-B (Buffer-A with no lysozyme and $0.3 \mathrm{mM}$ ATP) at $4{ }^{0} \mathrm{C}$. The column was washed with buffer- $\mathrm{B}+20 \mathrm{mM}$ imidazole and eluted the protein in buffer- $\mathrm{B}+250 \mathrm{mM}$ imidazole. The protein was loaded on Superdex $200^{\mathrm{TM}}$ (16/60) column (GE Healthcare Ltd), preequilibrated with buffer-B (50mM Tris-HCl pH 7.5, 15\% (v/v) Glycerol, 10 mM Arginine, $300 \mathrm{mM}$ $\mathrm{NaCl}, 10 \mathrm{mM} \mathrm{MgCl}$, $4 \mathrm{mM} \beta$-mercaptoethanol, $0.3 \mathrm{mM}$ ATP). The protein fractions were pooled and concentrated using 3kDa cutoff ultracentrifugal device (Millipore). The protein 
was analyzed on $12 \%$ SDS-PAGE and mass spectrometry. The protein concentration was determined using Bradford assay.

Site directed mutagenesis (Stratagene Ltd) was performed using pET23a-D2 plasmid as template. Forward and reverse primers were designed to generate the Q87A, T92A, S91A and K90A mutants of D2 domain (Table S1). PCR reaction was performed, followed by DpnI digestion and transformed all plasmids in E. coli DH5 $\alpha$ cells. Gene sequencing was performed on all four mutant plasmids to confirm the presence of correct mutation. All D2 mutant enzymes were overexpressed and purified using wild type D2 domain purification protocol.

\section{Preparation of wild type and mutant D3 domains}

The D3 domain gene encoding (349-580 residues) was amplified using M. tuberculosis $H 37 R v$ strain and cloned into $p E T-S U M O$ vector using TA cloning method. The resulting D3 gene plasmid was verified by gene sequencing. The D3 domain gene plasmid was transformed in E. coli BL21(DE3) cells (Kanamycin 150 $\mu \mathrm{g} / \mathrm{ml}$ ) and grew the single colony till $\mathrm{OD}_{600} \sim 0.6-0.7$ at $37{ }^{\circ} \mathrm{C}$. The cell culture was induced with $0.5 \mathrm{mM}$ IPTG at $12^{\circ} \mathrm{C}$ and grew for another $20 \mathrm{hr}$. The cell culture was harvested at $14,000 \mathrm{xg}$ and suspended the pellet in lysis buffer-A (50 mM Tris/ $\mathrm{HCl} \mathrm{pH} 7.5,300 \mathrm{mM} \mathrm{NaCl}, 4 \mathrm{mM} \beta-$ mercaptoethanol, 15\% Glycerol, 2 mM Benzamidine-hydrochloride, $1 \mathrm{mM}$ Phenylmethylsulfonyl fluoride, $10 \mathrm{mM} \mathrm{MgCl}$, $0.3 \mathrm{mM} \mathrm{ATP,} 0.2 \mathrm{mg} / \mathrm{ml}$ Lysozyme and $10 \mathrm{mM}$ Arginine). The cell lysate was homogenized, sonicated and centrifuged at $25,000 \mathrm{x}$ g at $4{ }^{0} \mathrm{C}$.

The supernatant was loaded on Ni-NTA column, preequilibrated with buffer-B (Buffer-A with no Lysozyme) and washed two times with buffer-B+20mM imidazole. The 
protein was eluted in buffer-B $+250 \mathrm{mM}$ imidazole. The $p E T S U M O-\mathrm{D} 3$ domain was loaded on Superdex $200^{\mathrm{TM}}$ (16/60) column pre-equilibrated with buffer-B (50mM Tris-HCl pH 7.5, $10 \%$ Glycerol, 300mM NaCl, 10mM Arginine, $2 \mathrm{mM} \beta$-mercaptoethanol, 0.3mM ATP and $10 \mathrm{mM} \mathrm{MgCl} 2$ ). The protein fractions were collected and analyzed on $12 \%$ SDS-PAGE.

For SUMO-tag cleavage, $p E T S U M O-D 3$ domain was mixed with SUMO protease in 1000:1 (w/w) ratio and dialyzed in buffer (50mM Tris- $\mathrm{HCl} \mathrm{pH} 8.0,0.2 \%$ Igepal and $1 \mathrm{mM}$ DTT) for $12 \mathrm{hr}$ at $4^{0} \mathrm{C}$. The reaction was stopped with $2 \mathrm{mM}$ PMSF and loaded on Superdex $200^{\mathrm{TM}}$ HiLoad (16/60) column, preequilibrated with buffer-B. The peak fraction containing cleaved D3 domain were pooled, concentrated and analyzed on 12\% SDS-PAGE.

Site directed mutagenesis (Stratagene) was performed using pETSUMO-D3 domain plasmid. Forward and reverse primers were designed to generate the five K382A, T383A, T384A, A574G and Y576A mutants of D3 domain plasmid (Table S1). The gene sequencing was performed on D3 mutant plasmids to confirm the correct mutation. All D3 domain mutants were overexpressed and purified by protocol used for wild type D3 purification.

\section{Preparation of EsxAB heterodimer}

The EsxA and EsxB proteins were overexpressed and purified as described in earlier paper [27]. In brief, the EsxA gene was cloned in pET21a vector and transformed in E. coli $B L 21(D E 3)$ cells having antibiotics (Ampicillin $100 \mu \mathrm{g} / \mathrm{ml}$ ). The EsxB gene was cloned in pET28a vector and transformed in E. coli BL21(DE3) cells having antibiotics (Kanamycin $50 \mu \mathrm{g} / \mathrm{ml}$ ). Single colony was inoculated in 31 luria bertani media with antibiotics and grew the cells at $37{ }^{0} \mathrm{C}$ till $\mathrm{OD}_{600} \sim 0.6-0.7$. The cells were induced with $1 \mathrm{mM}$ IPTG and grew for 
another $4 \mathrm{hr}$. The cells were harvested at $8000 \mathrm{rpm}$ for $3 \mathrm{~min}$. The EsxA and EsxB proteins overexpressed in soluble fraction of cells. For complex preparation, EsxA and EsxB proteins were mixed in 1:1 molar ratio and analyzed on native-PAGE for complex formation and purified using Superdex $200^{\mathrm{TM}}(16 / 60)$ Hiprep column.

\section{ATPase activity}

ATPase activity of full length EccCb1

For ATPase activity analysis, EccCb1 was dissolved in ATPase buffer containing (25mM Tris- $\left.\mathrm{HCl} \mathrm{pH} 7.5,10 \mathrm{mM} \mathrm{MgCl}_{2}\right) .0 .5 \mu \mathrm{Ci}$ of [ $\left.{ }^{\circledR}{ }^{32 \mathrm{P}}\right] \mathrm{ATP}$ was added in ATPase buffer and incubated at $37{ }^{\circ} \mathrm{C} .1 \mu \mathrm{l}$ of reaction mixture was spotted after every 15 min on TLC plate. The $0.5 \mathrm{M}$ formic acid and $0.5 \mathrm{M} \mathrm{LiCl}$ solution were used to develop TLC plate and dried at $37{ }^{0} \mathrm{C}$. The TLC plate was exposed to Fuji film BAS-MS 2025 imaging plate for $12 \mathrm{hr}$ and analyzed using Typhoon FLA 9500 (GE Healthcare Ltd). The background obtained from reaction buffer (having no EccCb1) was corrected from each measurement.

Colorimetric assay was performed to measure the EccCb1 ATPase activity using ATPase assay kit (Innova bioscience). The ATPase assay was performed at $37^{\circ} \mathrm{C}$ for 30 min with $1 \mu \mathrm{l}$ of EccCb1 $(2 \mu \mathrm{g})$ in $200 \mu \mathrm{l}$ of buffer (20mM HEPES pH 7.5, 150mM NaCl, $5 \%$ Glycerol, $2 \mathrm{mM} \beta$-mercaptoethanol, $10 \mathrm{mM} \mathrm{Mg}^{2+}$ and variable amount of ATP) in 96 well plate. The dye buffer containing $(120 \mathrm{mM}$ malachite green, $0.06 \%$ polyvinylalcohol, $6 \mathrm{mM}$ ammonium heptamolybdate, $4.2 \%$ sodium citrate) was added in the reaction buffer. After incubating for $15 \mathrm{~min}, 10 \mu \mathrm{l}$ solution from every reaction mixture was transferred in 96 well plates and absorbance at $630 \mathrm{~nm}$ was measured. Absorbance from reaction buffer (having no EccCb1) was subtracted from each data. Based on the absorbance from phosphate standard 
curve, the release of inorganic phosphate was measured. Each assay was performed three times and average ATPase activity was calculated. The kinetic parameters $\left(K_{m}, V_{\max }\right.$ and $\left.K_{c a t}\right)$ were calculated using Prism 6.0.2 program.

ATPase activity of EccCb1 was measured using $\left[\square^{32 \mathrm{P}}\right]$ ATP as substrate at different time intervals in presence of $\mathrm{Mg}^{2+}, \mathrm{Mn}^{2+}, \mathrm{Ca}^{2+}$, EDTA and high $\mathrm{NaCl}$ concentration. The EccCb1 was dialyzed in buffer $25 \mathrm{mM}$ Tris- $\mathrm{HCl} \mathrm{pH} 7.5$ for $12 \mathrm{hr}$ at $4^{\circ} \mathrm{C}$. The EccCb1 was added in reaction buffer $(25 \mathrm{mM}$ Tris- $\mathrm{HCl} \mathrm{pH} 7.5,150 \mathrm{mM} \mathrm{NaCl}, 5 \%$ Glycerol, $2 \mathrm{mM} \beta$ mercaptoethanol, $10 \mathrm{mM} \mathrm{MgCl} 2,1 \mathrm{mM}$ ATP and $0.5 \mu \mathrm{Ci}$ of $\left[\square^{32 \mathrm{P}}\right]$ ATP). The reaction mixture was kept at $37^{\circ} \mathrm{C}$ for $1 \mathrm{hr}$ and $1 \mu \mathrm{l}$ of solution was spotted on TLC plate, dried and developed using $0.5 \mathrm{M} \mathrm{LiCl}$ and $1 \mathrm{M}$ formic acid. The plate was dried, kept in IP for $12 \mathrm{hr}$ and scanned using Typhoon FLA 9500. Intensity of each spot was measured using Image J software [28]. Similar experiments were performed with $10 \mathrm{mM} \mathrm{Ca}^{2+}$ and $10 \mathrm{mM} \mathrm{Mn}^{2+}$ to examine their effects on EccCb1 ATPase activity.

\section{ATPase activity of wild type and mutant D2 domains}

ATPase activity of wild type and four D2 mutants were determined using calorimetric assay using ATPase kit obtained from INNOVA Biosciences Ltd. 1 gg of D2 domain was dissolved in $200 \mu$ of reaction buffer $(50 \mathrm{mM}$ Tris- $\mathrm{HCl} \mathrm{pH} 7.5,15 \%$ Glycerol, $10 \mathrm{mM} \mathrm{MgCl} 2,150$ $\mathrm{mM} \mathrm{NaCl}, 10 \mathrm{mM}$ Arginine) and incubated $10 \mathrm{~min}$ at $25{ }^{0} \mathrm{C}$ using various concentrations of ATP. $50 \mu 1$ of Malachite green reagent was mixed in reaction mixture and incubate for 30 min. The absorbance at $600 \mathrm{~nm}$ was measured and absorbance from reaction buffer (no D2 domain) was subtracted from each data. The released inorganic phosphate was estimated using standard absorbance curve based on $\mathrm{KH}_{2} \mathrm{PO}_{4}$ assay. All assays were performed three 
times and kinetic parameters $\left(K_{m}, V_{\max }\right.$ and $\left.K_{c a t}\right)$ were calculated using Prism 6.0.2 program (GraphPad Software). Similar protocol was used for ATPase activity analysis of four D2 mutant enzymes.

\section{ATPase activity of wild type and mutant D3 domains}

Calorimetric assay was performed for ATPase activity analysis of wild type and five D3 mutants using ATPase kit from INNOVA Biosciences Ltd. Initially, D3 domain was dialyzed in buffer (25 mM Tris-HCl pH 7.5, $0.5 \mathrm{mM} \beta$-mercaptoethanol, $150 \mathrm{mM} \mathrm{NaCl}, 5 \%$ Glycerol). $1 \mu \mathrm{M}$ of D3 domain was added in $200 \mu \mathrm{l}$ of reaction mixture $(50 \mathrm{mM}$ Tris- $\mathrm{HCl}$ $\mathrm{pH}$ 7.5, $15 \%$ Glycerol, $10 \mathrm{mM} \mathrm{MgCl} 2,150 \mathrm{mM} \mathrm{NaCl}, 10 \mathrm{mM}$ Arginine) from ATPase kit. The reaction mixture was incubated $30 \mathrm{~min}$ at $20{ }^{\circ} \mathrm{C}$ and $50 \mu \mathrm{l}$ of Pi color lock was added in each well. After 2 min, $20 \mu$ of stabilizer was added in each well, incubated for another 30 min and measured the absorbance at $630 \mathrm{~nm}$. All assays were performed three times and kinetic parameters $\left(K_{m}, V_{\max }\right.$ and $\left.K_{c a t}\right)$ were calculated using Prism 6.0.2 program (GraphPad Software). Similar protocol was used for ATPase activity measurement of all five D3 mutants.

\section{Small angle $\mathrm{X}$-ray scattering analysis}

Small angle X-ray scattering data on EccCb1 was collected using 1D Dectris Mythen detector at $10{ }^{\circ} \mathrm{C}$ using $0.154 \mathrm{~nm}$ wavelength at SAXSpace equipment at IMTECH, India (Table 1). $40 \mu \mathrm{l}$ of EccCb1 enzyme ( $5 \mathrm{mg} / \mathrm{ml})$ in 50mM Tris- $\mathrm{HCl} \mathrm{pH} 7.5,4 \mathrm{mM} \beta$ mercaptoethanol, $300 \mathrm{mM} \mathrm{NaCl}$ buffer was loaded on thermostated $1 \mathrm{~mm}$ quartz capillary and exposed $30 \mathrm{~min}$. Three frames were collected and averaged the data for protein and 
buffer. SAXSTreat program was used to correct the beam position, to subtract the buffer contribution and desmearing of scattering profile. The SAXS intensity profile was obtained as $\mathrm{I}(\mathrm{q}) \sim \mathrm{q}$ (momentum transfer vector). ATSAS suite of programs (version 3.0.1) [29] was used for all further data analysis.

The scattering profile of EccCb1 in solution was analyzed using Kratky plot (I(q)

$\mathrm{q}^{2}$ ). Guinier analysis was performed to obtain the $\mathrm{R}_{\mathrm{g}}$ (radius of gyration) of EccCb1 in solution. Using I(q) profile, $\mathrm{P}(\mathrm{r})$ curve and estimated parameters, the DAMMIF program of ATSAS suite [30] was used for ab initio shape determination using dummy atom model of EccCb1. DAMMIF program [30] generated the ten independent envelopes after aligning on inertial axes, comparison, clustering in family-based similarity and averaging. These de novo models were fitted with homology models of EccCb1 by PyMol based plugin SUPALM program [31].

\section{Biolayer interferometry analysis}

The interaction of wild type Rv3871 and its virulence factor (export arm of EsxAB heterodimer) was carried out using Forte Bio Octet Red 96 instrument at $30^{\circ} \mathrm{C}$ with 1000 rpm agitation. $50 \mu \mathrm{g} / \mathrm{ml}$ of Rv3871 and its mutants were individually immobilized on AR2G sensor and the experiment was carried out in GREINER 96 Solid black flat-bottom well plate in running buffer containing 20mM HEPES pH 7.5 and 150mM NaCl. Five different concentrations of virulence factor were prepared $(30 \mu \mathrm{M}$ to $15 \mu \mathrm{M}$ and $7.5 \mu \mathrm{M}$ to $3.8 \mu \mathrm{M}$ and $1.9 \mu \mathrm{M})$ in running buffer. Monitoring the interactions was conducted as $60 \mathrm{sec}$ for initial base line, $180 \mathrm{sec}$ association, $180 \mathrm{sec}$ dissociation and finally regeneration using glycine$\mathrm{HCl} \mathrm{pH}$ 3.0. Data analysis was done using Forte Bio Data Analysis 10 and 1:1 binding 
model was used for curve fitting and calculation of association constant, dissociation constant and equilibrium dissociation constant.

\section{Molecular modeling and dynamics simulation}

Homology modeling

The X-ray coordinates of EsxAB heterodimer were obtained from PDB 1WA8 (Solution structure of the CFP-10-ESAT-6 complex, major virulence determinants of pathogenic Mycobacteria) [32]. The EccCb1 model (1-591 residues) was obtained from I-TASSER server [33] using default parameters. The LOMETS program [34] used the EccCb1 sequence to identify the best structure templates from PDB database. The best template with high Zscore (high threading alignment score) was obtained from SPICKER program [35] after structural assembly simulation, and predicted the best models based on the C-score. The ITASSER server yielded the best EccCb1 model (23-591 residues) based T. cvurvata EccC crystal structure (PDB-4NH0). 
The crystal structure of D3 domain (PDB-6J19, 320-580 residues) was inserted in to obtained EccCb1 model based on PDB-4NH0 as template. The ATP $+\mathrm{Mg}^{2+}$ ligands were placed in D2 domain (23-327 residues) using PDB-4NH0 structure and in D3 domain (320580 residues) based on PDB-6J19 structure. Using EccCb1+peptide+ATP+Mg ${ }^{2+}$ crystal structure (PDB-6J19) as template, the EsxAB heterodimer was docked into EccCb1+ $\mathrm{ATP}+\mathrm{Mg}^{2+}$ complex using COOT program [36], which yielded the entire EccCb1+EsxAB+ $\mathrm{ATP}+\mathrm{Mg}^{2+}$ complex structure.

To improve EsxAB docking into D3 domain, induced fit docking module was used in GLIDE program [37] with 0.6-fold scaling on van der Waals radii and XP (extra precision scoring function) for docking analysis. All residues within $6 \AA$ radii of EsxAB substrate were refined for prime site optimization. 10,000 cycles of energy minimization and 10,000 cycles of scoring were performed using Glidestone module of Glide program, which yielded the best EccCb1+EsxAB+ATP+Mg ${ }^{2+}$ complex model. The best complex model was selected based on energy, interaction, buried surface area and lowest IFD.

The best EccCb1+EsxAB+ATP+Mg ${ }^{2+}$ model was given as input in SymmDock server [38] and generated the EccCb1+EsxAB+ATP+Mg ${ }^{2+}$ hexamer using 6-fold symmetry as constraint. The best hexameric complex was selected based on scoring function and given as input in SymmDock server using 2 fold symmetry, which yielded the cylindrical dodecameric structure of EccCb1+EsxAB+ATP+ $\mathrm{Mg}^{2+}$ complex. 50,000 steps of steepest descent minimization was performed on obtained EccCb1+EsxAB+ATP+ $\mathrm{Mg}^{2+}$ dodecamer using GROMACS program [39]. Structural validation on obtained dodecameric complex PROCHECK program [40] program was structural validation yielded the good stereochemistry of obtained model. 
The MultAln [41] and ESPript 3.0 [42] programs were used for multiple sequence alignment of EccCb1 model against T. curvata EccC amd G. Dentrificans EssC sequences, best structural homologs identified by I-TASSER server. Structural superposition of EccCb1 against both enzymes and fitting into observed SAXS envelope were performed using CHIMERA program [43].

Dynamics simulation on Apo, ATP $+\mathrm{Mg}^{2+}$ and $A T P+M g^{2+}+E s x A B$ bound EccCbl dodecamer

The dynamics simulation on (i) apo EccCb1 dodecamer (ii) EccCb1+ATP+Mg ${ }^{2+}$ dodecamer and (iv) EccCb1+EsxAB+ATP+ $\mathrm{Mg}^{2+}$ dodecamer were performed using in GROMACS 2020.1-MODIFIED program using CHARMM36 all atom force field [44] and TIP3P water molecules parameters [45]. Details of all parameters used in dynamics simulation of EccCb1 models are given in Table 4. The EccCb1 models were kept in cubic box extended by 1.0 nm from protein surface and solvated using explicit SPC water molecules. Counter ions were added to neutralize the charge of the system. Before dynamics simulation, 50,000 steps of steepest descents energy minimization were performed on EccCb1 models. The particle-mesh Ewald (PME) method was used for electrostatic interactions having following parameters e. g. grid spacing $1.0 \AA$, cutoff $10 \AA$, relative tolerance of $10^{-6}$ and $10 \AA$ switching for Lennard-Jones interactions. LINCS algorithm [46] was used for constraining bonds involved in hydrogen bonding.

100 ps of NVT equilibration was performed after heating the whole system at $300 \mathrm{~K}$, followed by 100 ps NPT equilibration. Finally, dynamics simulation was performed under NPT condition with $\mathrm{V}$ - rescale (modified Berendsen thermostat) coupling using Gromacs 
10.12 version program. The 1.0 coulomb cut off was applied and 2 fs time step was used in dynamics simulation analysis. After every 4 ps, the coordinates were saved for MD trajectory analysis. The pressure was maintained by Parrinello-rahman pressure coupling constant and coulomb cut off was applied during temperature coupling. The Plot2X program [47] for trajectories analysis, PyMoL [48] and CHIMERA programs for structure visualization.

\section{Conflict of interest}

The authors have no conflict of interest.

\section{Acknowledgements}

We acknowledge the financial support from Department of Science and Technology, New Delhi DST-project (No. DST-EMR/2016/000867) to conduct the current research work.

Current work was also partially supported by UGC-SAP grant, UGC-resource networking grant, DST-PURSE grant. We acknowledge the UGC, India for Senior Research Fellowship support to Arkita. The staff from CIF facility of SLS, JNU is gratefully acknowledged. We acknowledge the help from Dr. Ashish and other staff at IMTECH facility for SAXS data collection.

\section{Author's contributions}

Arkita cloned, expressed, purified the wild type and mutant EccCb1 proteins, She performed all ATPase assays and binding analysis with native and mutant proteins. Ajay K. Saxena performed all small angle X-ray scattering structure analysis, molecular modeling, docking 
bioRxiv preprint doi: https://doi.org/10.1101/2021.05.31.446396; this version posted May 31, 2021. The copyright holder for this preprint (which was not certified by peer review) is the author/funder. All rights reserved. No reuse allowed without permission.

and dynamic simulation of all EccCb1 complexes. Ajay K. Saxena conceived, designed the strategies, analyzed the whole data and wrote the entire manuscript. 


\section{References}

1. MacGurn JA \& Cox JS (2007) A genetic screen for Mycobacterium tuberculosis mutants defective for phagosome maturation arrest identifies components of the ESX-1 secretion system. Infect Immun. 75(6), 266802678.

2. Simeone R, Bottai D, Brosch R (2009) ESX/type VII secretion systems and their role in host-pathogen interaction. Curr Opin Microbiol. 12(1), 4-10.

3. Famelis N, Rivera-Calzada A, Degliesposti G et. al. (2019) Architecture of the mycobacterial type VII secretion system. Nature, 576(7786), 321-325.

4. Poweleit N, Czudnochowski N, Nakagawa R, Trinidad DD, Murphy KC, Sassetti CM \& Rosenberg OS (2019) The structure of the endogenous ESX-3 secretion system. Elife, 8, e52983.

5. Beckham KS, Ciccarelli L, Bunduc CM et. al. (2017) Structure of the mycobacterial ESX5 type VII secretion system membrane complex by single-particle analysis. Nat Microbiol. 2, 17047.

6. Abdallah AM, Gey van Pittius NC, Champion P et al. (2007) Type VII secretion-mycobacteria show the way. Nat Rev Microbiol. 5(11),883-891.

7. Massey TH, Mercogliano CP, Yates J, Sherratt DJ \& Löwe J (2006) Double-stranded DNA translocation: structure and mechanism of hexamericFtsK. Mol Cell. 23(4), 457-469.

8. Erzberger JP \& Berger JM (2006) Evolutionary relationships and structural mechanisms of AAA+ proteins. Annu Rev BiophysBiomol Struct. 35, 93-114.

9. Ahmadian MR, Stege P, Scheffzek K \& Wittinghofer A (1997) Confirmation of the arginine-finger hypothesis for the GAP-stimulated GTP-hydrolysis reaction of Ras. Nat Struct Biol. 4(9), 686-689.

10. Rosenberg OS, Dovala D, Li X et al. (2015) Substrates Control Multimerization and Activation of the Multi-Domain ATPase Motor of Type VII Secretion. Cell,161(3), 501-512.

11. Zoltner M, Ng WM, Money JJ, et al. (2016) EssC: domain structures inform on the elusive translocation channel in the Type VII secretion system. Biochem J. 473(13), 19411952.

12.Bitter W, Houben EN, Bottai D et al. (2009) Systematic genetic nomenclature for type VII secretion systems. PLoSPathog. 5(10), e1000507.

13. Pallen MJ (2002) The ESAT-6/WXG100 superfamily -- and a new Gram-positive secretion system? Trends Microbiol. 10(5),209-212. 
14. Champion PA, Champion MM, Manzanillo P \& Cox JS (2009) ESX-1 secreted virulence factors are recognized by multiple cytosolic AAA ATPases in pathogenic mycobacteria. Mol Microbiol. 73(5), 950-962.

15. Fortune SM, Jaeger A, Sarracino DA, et al. (2005) Mutually dependent secretion of proteins required for mycobacterial virulence. Proc Natl Acad Sci U S A. 102(30), 1067610681.

16. Wang S, Zhou K, Yang X, Zhang B, Zhao Y, Xiao Y, Yang X, Yang H, Guddat LW, Li J \& Rao Z (2020) Structural insights into substrate recognition by the type VII secretion system. Prot. Cell, 11(2), 124-137.

17. Mietrach N, Damián-Aparicio D, Mielich-Süss B, Lopez D \& Geibel S (2020) Substrate Interaction with the EssC Coupling Protein of the Type VIIb Secretion System. J.Bacteriol. 202(7), e00646-19.

18. Swift ML (1997) GraphPad Prism, data analysis and scientific graphing. J Chem Inf Comput Sci.37, 411-412.

19. Svergun DI, Barberato C and Koch MHJ (1995) CRYSOL - a Program to Evaluate Xray Solution Scattering of Biological Macromolecules from Atomic Coordinates. J. Appl. Cryst. 28, 768-773.

20. Crooks GE, Hon G, Chandonia JM \& Brenner SE (2004) WebLogo: a sequence logo generator. Genome Res. 14(6),1188-1190.

21. Wallace AC \& Laskowski RA \& Thornton JM (1995) LIGPLOT: a program to generate schematic diagrams of protein-ligand interactions. Protein Eng. 8(2),127-134.

22. Laskowski R A, Jabłońska J, Pravda L, Vařeková R S \& Thornton J M (2018). PDBsum: Structural summaries of PDB entries. Prot. Sci. 27, 129-134.

23. Redzej A, Ukleja M, Connery S, et al. (2017) Structure of a VirD4 coupling protein bound to a VirB type IV secretion machinery. EMBO J. 36(20),3080-3095.

24. Massey TH, Mercogliano CP, Yates J, Sherratt DJ \& Löwe J. (2006) Double-stranded DNA translocation: structure and mechanism of hexameric FtsK. Mol Cell 23(4),457-69.

25. Fletcher RJ, Bishop BE, Leon RP, Sclafani RA, Ogata CM \& Chen XS (2003) The structure and function of MCM from archaealM. Thermoautotrophicum. Nat Struct Biol.10, $160-167$.

26. Li D, Zhao R, Lilyestrom W, Gai D, Zhang R, DeCaprio JA, Fanning E, Jochimiak A, Szakonyi G \& Chen XS (2003) Structure of the replicative helicase of the oncoprotein SV40 large tumour antigen. Nature 423, 512-518. 
27. Kulshrestha A, Gupta A, Verma N, Sharma SK, Tyagi AK \& Chaudhary VK (2005) Expression and purification of recombinant antigens of Mycobacterium tuberculosis for application in serodiagnosis, Prot. Express \& Purifi. 44(1), 75-85.

28. Abramoff MD, Magalhães PJR \& Sunanda J (2004) Biophotonics inter. 11(7), 36-42.

29. Franke D, Petoukhov MV, Konarev PV, Panjkovich A, Tuukkanen A, Mertens HDT, Kikhney AG, Hajizadeh NR, Franklin JM, Jeffries CM \& Svergun DI (2017) ATSAS 2.8: a comprehensive data analysis suite for small-angle scattering from macromolecular solutions. J Appl Cryst. 50, 1212-1225.

30. Franke D \& Svergun, DI (2009) DAMMIF, a program for rapid ab-initio shape determination in small-angle scattering. J. Appl. Cryst., 42, 342-346.

31. Konarev PV, Petoukhov MV \& Svergun DI (2016) Rapid automated superposition of shapes and macromolecular models using spherical harmonics._J Appl Cryst._49, 953-960.

32. Renshaw PS, Lightbody KL, Veverka V, et al. (2005) Structure and function of the complex formed by the tuberculosis virulence factors CFP-10 and ESAT-6. EMBO J. 24(14), 2491-2498.

33. Zhang Y (2008) I-TASSER server for protein 3D structure prediction. BMC Bioinformatics. 9, 40.

34. Wu S \& Zhang Y (2007) LOMETS: a local meta-threading-server for protein structure prediction. Nucleic Acids Res. 35(10), 3375-3382.

35. Zhang Y \& Skolnick J (2004) SPICKER: a clustering approach to identify near-native protein folds. J Comput Chem. 25(6), 865-871.

36. 33. Emsley P \& Cowtan K. (2004) Coot, model-building tools for molecular graphics. Acta Crystallogr D Biol Crystallogr. 60(Pt 12 Pt 1), $2126 \square 2132$.

37. Friesner RA, Banks JL, Murphy RB et al. (2004) Glide: a new approach for rapid, accurate docking and scoring. 1. Method and assessment of docking accuracy. J Med Chem. 47(7), 1739-1749.

38. Schneidman-Duhovny D, Inbar Y, Nussinov R, Wolfson HJ (2005) PatchDock and SymmDock: servers for rigid and symmetric docking. Nucleic Acids Res. 2005 33(Web Server issue), W363-367.

39.Abraham MJ, Murtola T, Schulz R, Pa'1l S, Smith JC, Hess B \& Lindahl E (2015) GROMACS: high performance molecular simulations through multi-level parallelism from laptops to supercomputers. SoftwareX 1-2,19-25.

40. Laskowski RA, MacArthur MW, Moss DS \& Thornton JM (1993)

PROCHECK - aprogram to check the stereochemical quality of protein structures. $J$ 
AppCryst.26: 283-291.

41. Corpet F (1988) Multiple sequence alignment with hierarchical clustering. Nucleic Acids Res. 16(22),10881-10890.

42. Gouet P, Robert X \& Courcelle E (2003) ESPript/ENDscript: Extracting and rendering sequence and 3D information from atomic structures of proteins. Nucleic Acids Res.31(13), $3320-3323$.

43. Pettersen EF, Goddard TD, Huang CC, et al. (2004) UCSF Chimera--a visualization system for exploratory research and analysis. J Comput Chem. 25(13),1605-1612.

44. Huang J \& MacKerell ADJr. (2013) CHARMM36 all-atom additive protein force field, validation based on comparison to NMR data. J Comput Chem. 34(25), 2135-2145.

45. Nayar D, Agarwal M \& Chakravarty C (2011) Comparison of Tetrahedral Order, Liquid State Anomalies, and Hydration Behavior of mTIP3P and TIP4P Water Models. J Chem Theory Comput. 7(10),3354-3367.

46. Hess B, Bekker H, Berendsen HJC \& Fraaije J (1997) GEM. LINCS: A linear constraint solver for molecular simulations. J Comput Chem.1472, 1463-1472.

47. Kalesinskas L, Cudone E, Fofanov Y \& Putonti C (2018) S-plot2: Rapid Visual and Statistical Analysis of Genomic Sequences. EvolBioinform Online.14,11769343-18797354.

48. Rigsby RE \& Parker AB (2016) Using the PyMOL application to reinforce visual understanding of protein structure. Biochem Mol Biol Educ. 44(5),433-437. 
Table 1. Kinetic parameters of full length EccCb1 and its D2 \& D3 domains.

\begin{tabular}{|l|l|l|l|l|l|}
\hline Protein & \multicolumn{1}{|c|}{$\begin{array}{c}V_{\max } \\
\mu \mathrm{M} / \mathrm{min} / \mathrm{mg}\end{array}$} & $\begin{array}{c}K_{m} \\
\mathrm{mM}\end{array}$ & $\begin{array}{c}k_{\text {cat }} \\
\mathrm{min}^{-1}\end{array}$ & $\begin{array}{c}k_{c a} / K_{m} \\
\mu \mathrm{M}^{-1} \mathrm{~min}^{-1}\end{array}$ & $\begin{array}{c}\text { of } \\
\text { activity } \\
\text { observed }\end{array}$ \\
\hline $\begin{array}{l}\text { Full length } \\
\text { EccCb1 }\end{array}$ & $\mathbf{9 . 2 8}$ & $\mathbf{0 . 0 2} \pm \mathbf{0 . 0 1}$ & $\mathbf{0 . 3 1} \pm \mathbf{0 . 0 5}$ & $\mathbf{0 . 0 2 0} \pm \mathbf{0 . 0 0 5}$ & \\
\hline D2 domain & $\mathbf{6 3 . 7 9}$ & $\mathbf{0 . 0 3} \pm \mathbf{0 . 0 1}$ & $\mathbf{2 . 1 3} \pm \mathbf{0 . 1 0}$ & $\mathbf{0 . 0 7 0} \pm \mathbf{0 . 0 2 0}$ & $\mathbf{1 0 0 \%}$ \\
\hline D2-Q87A & 39.88 & $0.05 \pm 0.01$ & $1.33 \pm 0.08$ & $0.030 \pm 0.007$ & $44.1 \%$ \\
\hline D2-K90A & 23.92 & $0.20 \pm 0.06$ & $0.80 \pm 0.13$ & $0.004 \pm 0.002$ & $6.1 \%$ \\
\hline D2-S91A & 45.22 & $0.05 \pm 0.02$ & $1.15 \pm 0.13$ & $0.030 \pm 0.008$ & $42.2 \%$ \\
\hline D2-T92A & 65.22 & $0.19 \pm 0.10$ & $2.18 \pm 0.60$ & $0.010 \pm 0.006$ & $17.7 \%$ \\
\hline D3 domain & $\mathbf{5 2 . 8 9}$ & $\mathbf{0 . 0 6} \pm \mathbf{0 . 0 3}$ & $\mathbf{1 . 7 6} \pm \mathbf{0 . 2 7}$ & $\mathbf{0 . 0 3 0} \pm \mathbf{0 . 0 0 8}$ & $\mathbf{1 0 0 \%}$ \\
\hline D3- K382A & 17.49 & $0.40 \pm 0.28$ & $0.60 \pm 0.10$ & $0.002 \pm 0.001$ & $6.6 \%$ \\
\hline D3- T383A & 26.34 & $0.30 \pm 0.20$ & $0.88 \pm 0.50$ & $0.003 \pm 0.002$ & $10.3 \%$ \\
\hline D3- T384A & 41.70 & $0.40 \pm 0.10$ & $1.40 \pm 1.00$ & $0.004 \pm 0.001$ & $12.7 \%$ \\
\hline D3-A574G & 46.76 & $0.20 \pm 0.08$ & $1.56 \pm 0.35$ & $0.008 \pm 0.004$ & $26.5 \%$ \\
\hline D3- Y576A & 45.21 & $0.20 \pm 0.08$ & $1.51 \pm 0.30$ & $0.008 \pm 0.004$ & $24.8 \%$ \\
\hline
\end{tabular}


Table 2. SAXS data collection and de novo structure analysis of EccCb1

Instruments/programs data analysis

\section{SAXS Data Acquisition}

Instrument

Collimation

Wavelength (nm)

q range $(1 / \mathrm{nm})$

Exposure time

Protein Concentration

Temperature

\section{Programs}

Data Collection

Beam position correction

Buffer subtraction

Desmearing

\section{Data Analysis}

All parameter analysis

Shape Restoration

Inertial Alignment

Theoretical SAXS calculationCRYSOL

3D Graphics

PyMol, UCSF Chimera

Guinier based $\mathrm{R}_{\mathrm{g}}, \mathrm{M}_{\mathrm{w}}$

$\mathrm{P}(\mathrm{r})$ based $\mathrm{D}_{\max }, \mathrm{R}_{\mathrm{g}}$

NSD of models

$6.87 \mathrm{~nm}, 717.6 \mathrm{kDa}$

$20.14 \mathrm{~nm}, 6.86 \mathrm{~nm}$,

Resolution of ensemble

$0.883 \pm 0.132$

$9.7 \pm 0.7 \mathrm{~nm}$

SAXSDrive

SAXSTreat

SAXSQuant

SAXSQuant

Estimation

Dummy residue model

DAMAVER for Averaging

Guinier Analysis, Distance Distribution, Molecular Weight

10 models with online DAMMIF

DAMCLUST for clustering into families

Calculated $\chi^{2}$ of theoretical SAXS vs. Experimental Data

EccCb1 model $\quad 0.85$

Molmap Generation Scale 20 for DAMFILT and DAMAVER 
Table 3. Binding analysis between EccCb1 and EsxAB substrate using Biolayer interferometry $\left(K_{D}\right.$ is affinity constant, $K_{\text {on }}$ is association constant, and $K_{\text {off }}$ is dissociation constant).

\begin{tabular}{llcccc}
\hline Immobilized & Ligand & $\begin{array}{c}\text { ATP } \\
(\mathrm{mM})\end{array}$ & $\begin{array}{c}K_{\text {on }} \\
\left(10^{5} \mathrm{M}^{-1} \mathrm{~s}^{-1}\right)\end{array}$ & $\begin{array}{c}K_{\text {off }} \\
\left(10^{-3} \mathrm{~s}^{-1}\right)\end{array}$ & $\begin{array}{c}K_{D} \\
(\mathrm{~nm})\end{array}$ \\
\hline EccCb1 & EsxAB & 0.02 & $5.28 \pm 1.58$ & $6.03 \pm 0.07$ & $11.4 \pm 3.4$ \\
EccCb1 & EsxAB & - & $2.22 \pm 0.43$ & $7.16 \pm 0.12$ & $34.3 \pm 6.7$ \\
\hline
\end{tabular}


Table 4. Details of various parameters used in dynamic simulation on EccCb1 dodecamer in three different environments.

\begin{tabular}{llll}
\hline Models & Apo & ATP $+\mathrm{Mg}^{2+}$ & $\mathrm{ATP}+\mathrm{Mg}^{2+}+\mathrm{EsxAB}^{2+}$ \\
\hline Protein atoms & 50,814 & 50,814 & 50,814 \\
Water/Na & & $76,649 / 54$ & $137,446 / 114$ \\
Ligand atoms & $114,608 / 30$ & 24 & $24 / 8640$ \\
Minimization (Steep) & 50,000 & 50,000 & 50,0000 \\
NVT equilibration & $100 \mathrm{ps}$ & $100 \mathrm{ps}$ & $100 \mathrm{ps}$ \\
NPT equilibration & $100 \mathrm{ps}$ & $100 \mathrm{ps}$ & $100 \mathrm{ps}$ \\
Time steps (fs) & 2 & 2 & 2 \\
No. of steps & 500,000 & 500,000 & 500,000 \\
Simulation (ns) & 1 & 1 & 1 \\
\hline
\end{tabular}




\section{Figure's legends}

Fig. 1. Purification and ATPase activity of EccCb1 enzyme. A, Schematic diagram of full length EccCb1 (1-591 residues), consists of D2 domain (35-316 residues, blue) and D3 domain (349-580 residues, cyan). The 84-91 residues of D2 domain and 376-383 residues of D3 domain are involved in ATPase activity and highly conserved in Ftsk/SpoEIII family of enzymes. B, Elution profile of cleaved EccCb1 from Superdex ${ }^{\mathrm{TM}}$ 200(16/60) column. The protein eluted as oligomer (based on molecular mass standard, inset) and appeared as single band on SDS-PAGE (inset). C, Time dependent ATP hydrolysis of EccCb1 enzyme. Inset shows the radioactivity profile of the ATP hydrolysis. D, ATPase activity of EccCb1 enzyme using malachite green assay and $K_{m}, k_{c a t}$ and $V_{\max }$ parameters were obtained using Graph Pad software. E, Percentage of ATP hydrolysis by EccCb1 in presence of $10 \mathrm{mM}$ EDTA, $500 \mathrm{mM} \mathrm{NaCl}$ and $1 \mathrm{mM} \mathrm{MgCl}$. Inset shows the radioactivity profile of ATP hydrolysis. F, Percentage of ATP hydrolysis by EccCb1 in presence of different divalent cations. Inset shows the radioactivity profile of ATP hydrolysis

Fig 2. Small angle X-ray scattering analysis of EccCb1 enzyme. A, Experimental SAXS intensity profile of EccCb1. Inset shows the linear fit to Guinier region of measured data. B, Guinier plot in low q region showing linear agreement between experimental data (blue crosses) and Guinier equation (blue line) and further confirmed by low residuals within boundaries. C, Inter-atomic pairwise distribution function, P(r), calculated by GNOM program, which indicates the frequency distributions of interatomic vectors in predominant scattering species. At $\mathrm{D}_{\max } \sim 20 \AA$, the $\mathrm{P}(\mathrm{r})$ function approaches zero smoothly. D-E, 0 and $90^{0}$ views of double hexameric ring of EccCb1 fitted in molecular envelope generated by $a b$ 
initio SAXS modeling. F-G, 0 and $90^{\circ}$ views of double hexameric ring of EccCb1 $\left(180^{\circ}\right.$ view of Fig. D-E). H, Fitting of experimental scattering data (cyan) with theoretical scattering curve (red) obtained from 10 ab initio models generated by DAMMIF program. To generate envelope, DAMAVER superimposed all 10 models, averaged and filtered the final model to generate envelope, as shown in Fig. 5D-G. The mean normalized spatial discrepancy (NSD) of $0.883 \pm 0.132$ observed for 10 aligned models.

Fig. 3. Purification and ATPase activity of wild and mutant D2 domains. A, Web logo diagram of ATPase motif of D2 domain aligned with Ftsk/SpoEIII motif of 80 EccC sequences. B, Elution profile of wild and four mutant D2 domains obtained from Superdex $200^{\mathrm{TM}}$ (16/60) column and eluted as dimer from size exclusion column. C, LigPlot analysis of $\mathrm{D} 2+\mathrm{ATP}+\mathrm{Mg}^{2+}$ complex showing the interactions between ligand and D2 domain. ATP is shown in maroon, $\mathrm{Mg}^{2+}$ in green and all interacting residues in red. D, Michaelis-Menten plots showing the wild and four point mutant (Q87A, K90A, S91A, T92A) D2 domains.

Fig. 4. Purification and ATPase activity analysis of wild and mutant D3 domains. A, Web logo diagram of ATPase motif of D3 domain aligned with Ftsk/SpoEIII motif of 80 EccC sequences. B, Elution profile of wild type and five mutant D3 domains obtained from Superdex ${ }^{\mathrm{TM}} 200(16 / 60)$ column. The D3 domain eluted as dimer (based on molecular mass standard, inset) and protein appeared as single band on SDS-PAGE (inset) C, LigPlot analysis showing the interactions between $\mathrm{ATP}+\mathrm{Mg}^{2+}$ and D3 domain. The ATP is shown in maroon, $\mathrm{Mg}^{2+}$ in green and D3 residues in red. D, Michaelis-Menten plots showing the ATP 
hydrolysis profile of wild D3 domain and its K382A, T383A, T384A, A574A, Y576A mutants.

\section{Fig. 5. EsxAB substrate binding analysis using wild type and ATP bound EccCb1}

enzyme. A, Electrostatic surface diagram of EccCb1 hexamer contoured at $\pm 10 \mathrm{kT}$. The EsxA (cyan) and EsxB (orange) bind to partially positively charged groove at C-terminal of D3 domain. The central cavity of EccCb1 is highly negative. B, The EccCb1+ATP+ $\mathrm{Mg}^{2+}+$ EsxAB complex model showing the D3 domain pocket involved in binding to export arm of EsxAB virulence protein. $\mathbf{C}$, showing the hydrogen bonds and vander waals interactions between EccCb1 and EsxB virulence protein. D, Sensogram showing the interaction between EsxAB heterodimer and EccCb1 in absence of ATP. E, Sensogram showing the interaction between Esx AB heterodimer and EccCb1 in presence of $0.3 \mathrm{mM}$ ATP. The $K_{D}$ represents the binding affinity, $K_{o n}$ is the association constant and $K_{\text {off }}$ is the dissociation constant.

Fig. 6. Sequence alignment, molecular modeling and comparative structure analysis of EccCb1 enzyme. A, Sequence alignment of EccCb1 (Cyam) with two structural homologs e. g. T. curvata EccC enzyme (PDB - 4NH0, red) and D, EccC enzyme (PDB- 4YVA, blue)) using MultiAln and Espript 5 programs. Semi conserved residues are shown in red letters and highly conserved in red shade. The EccCb1 residues involved in ATP+ $\mathrm{Mg}^{2+}$ binding are shown in (\#) above sequence alignment and EsxB binding in (*) below the sequence alignment. The $\beta$-strands are shown in black arrow and $\alpha$-helices in spiral. $\mathbf{B}$, Superposition of T. curvata $\mathrm{EccC}(\mathrm{PDB}=4 \mathrm{NH}$, cyan) and D. EccC (PDB- 4LYA, green) 
structures on EccCb1+ATP $+\mathrm{Mg}^{2+}$ structure (red) using PyMol program. C, Superposition of D2 domain on D3 domain. D2 domain is shown in orange and D3 domain in cyan. D, The $\mathrm{EccCb} 1+\mathrm{ATP}+\mathrm{Mg}^{2+}+\mathrm{Esx} \mathrm{AB}$ hexameric dimer viewed along helical axis. Each complex is shown with red and cyan colors respectively. ATP is shown in yellow and $\mathrm{Mg}^{2+}$ in green colors respectively.

\section{Fig. 7. EccCb1 enzyme dynamics involved in $\mathrm{ATP}+\mathrm{Mg}^{2+}$ and EsxAB substrate}

recognition. A, Superposition of the simulated apo EccCb1 dodecamer (cyan) on starting EccCb1 dodecamer (grey). B, Superposition of simulated EccCb1+ATP $+\mathrm{Mg}^{2+}$ dodecamer (red) on starting dodecamer (grey). C, Superposition of simulated EccCb1+ EsxAB $+\mathrm{ATP}+\mathrm{Mg}^{2+}$ dodecamer (blue) on starting dodecamer (grey). D, RMSD of the EccCb1 models obtained during 1 ns dynamics simulation. E, Rg value of EccCb1 models obtained during 1 ns simulation. F, Plot showing the RMSF value of backbone $\mathrm{C} \alpha$ atoms of EccCb1 models. 


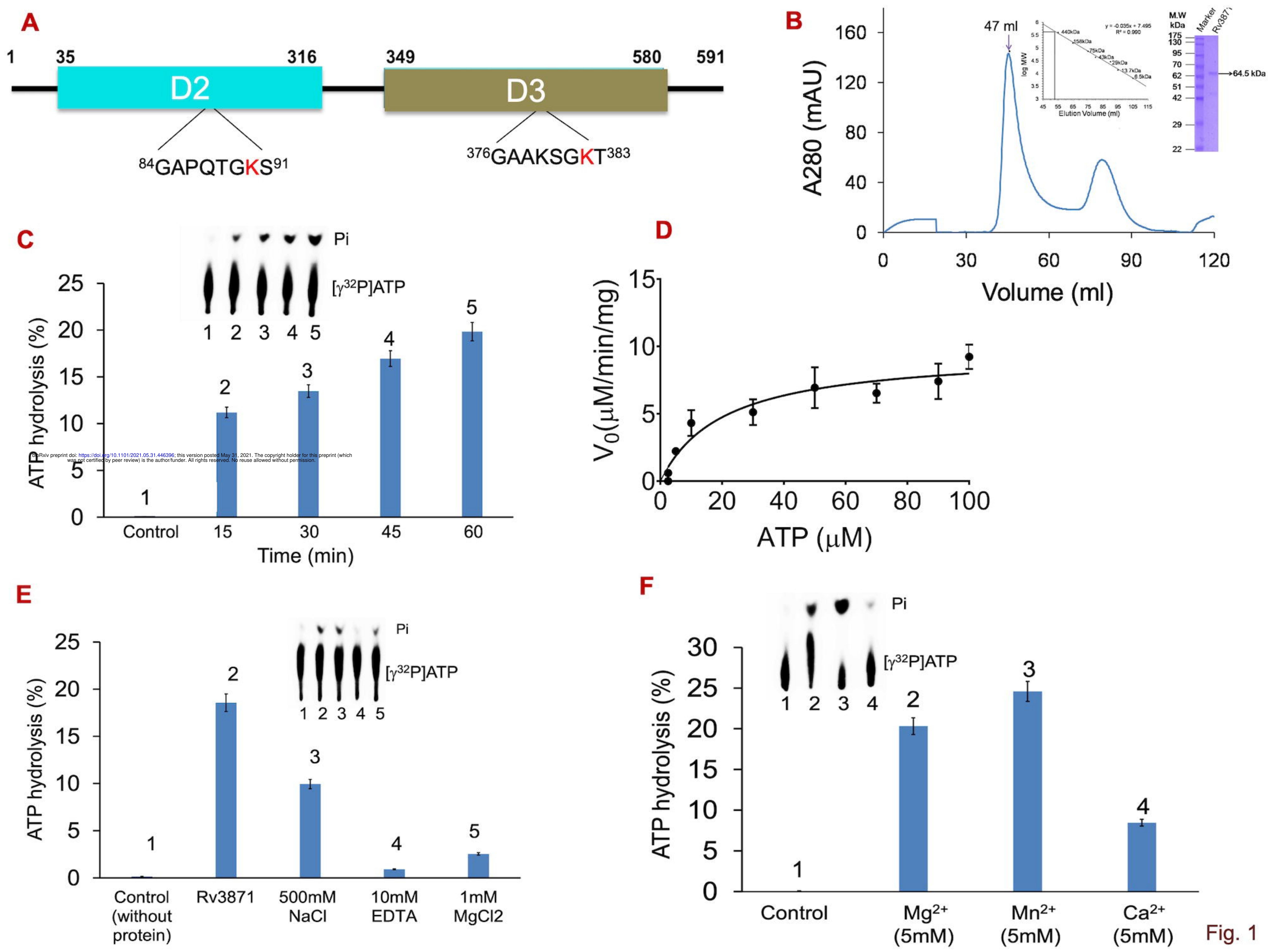


A Experimental scattering curve
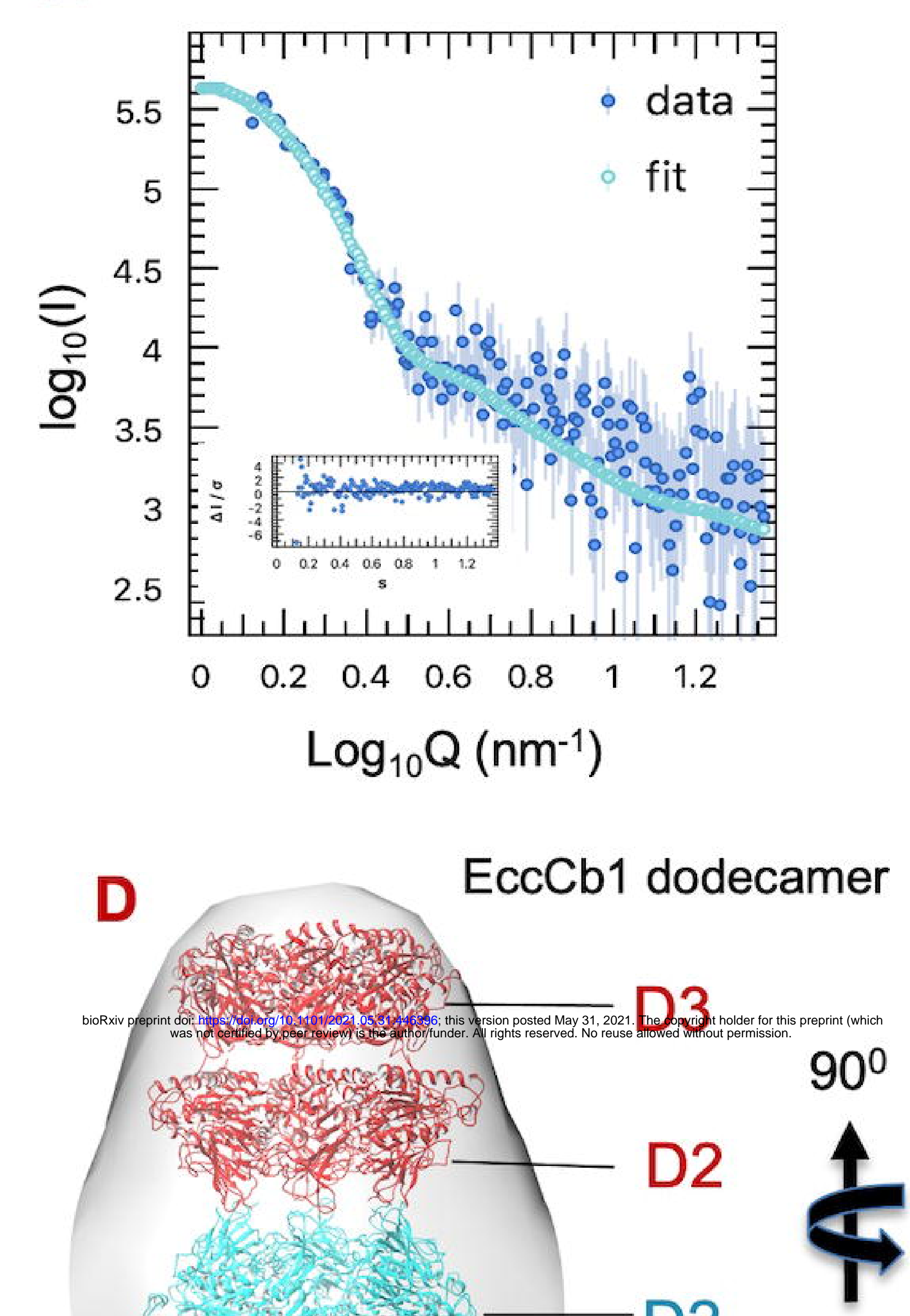

Raving

D3

F
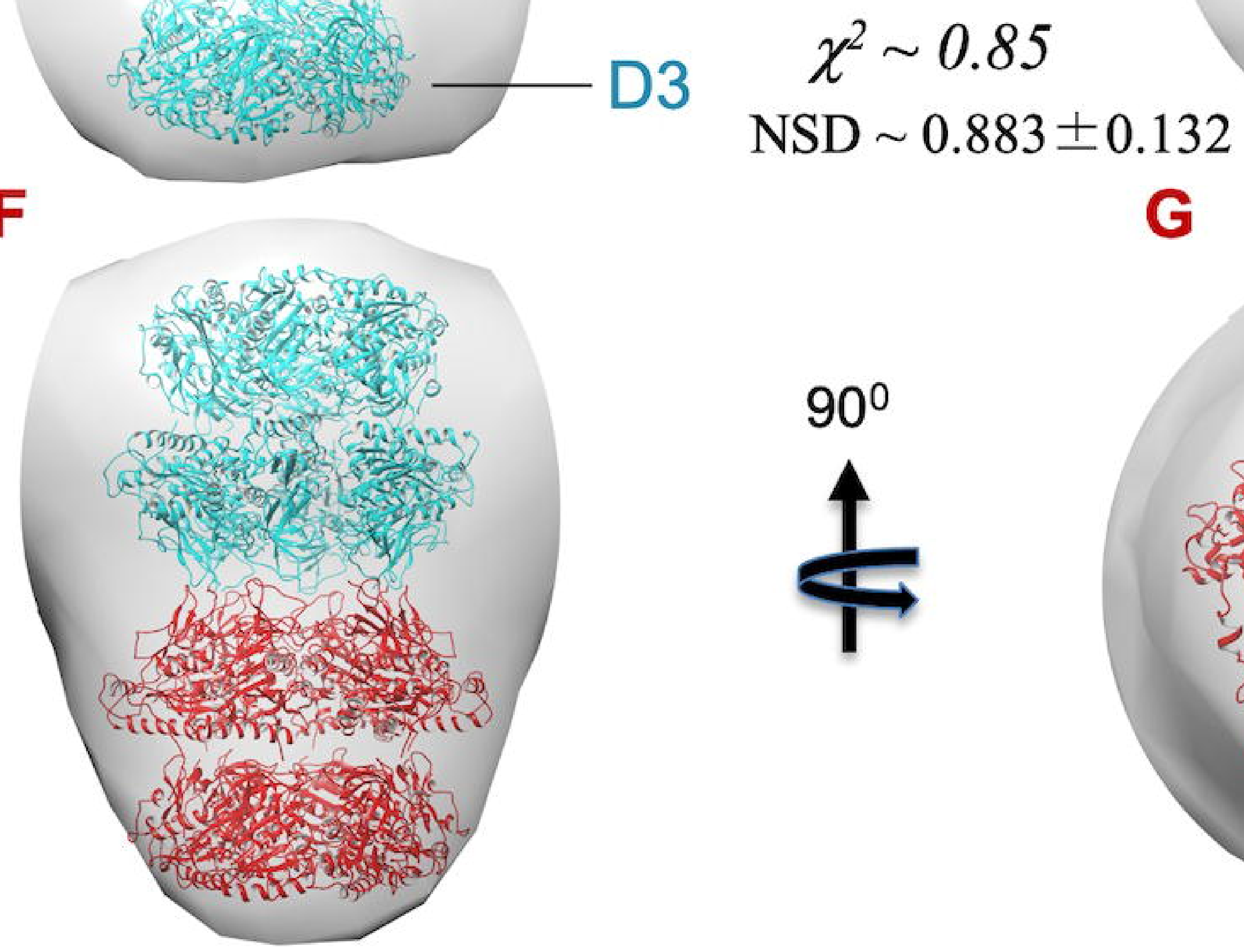
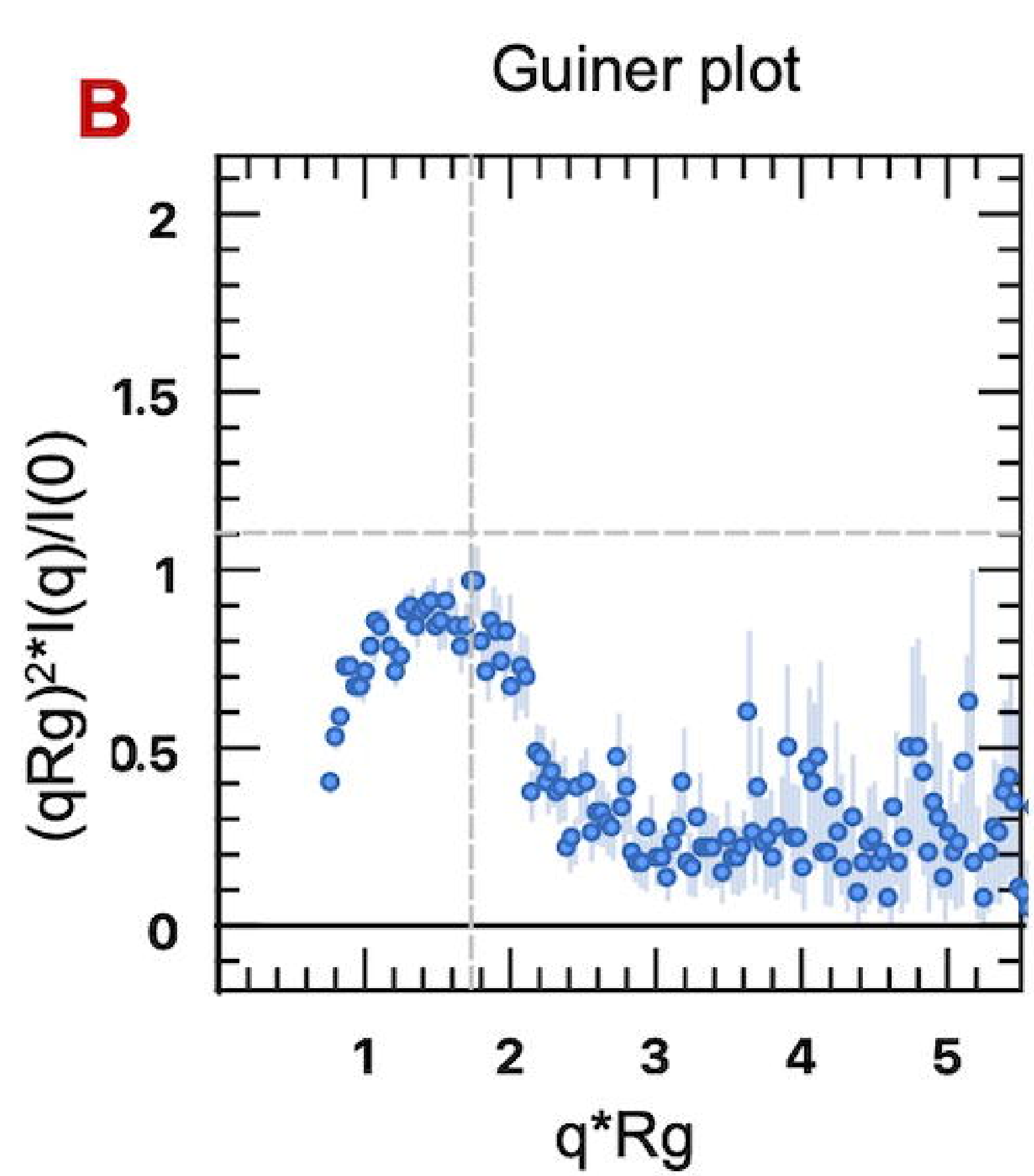

E

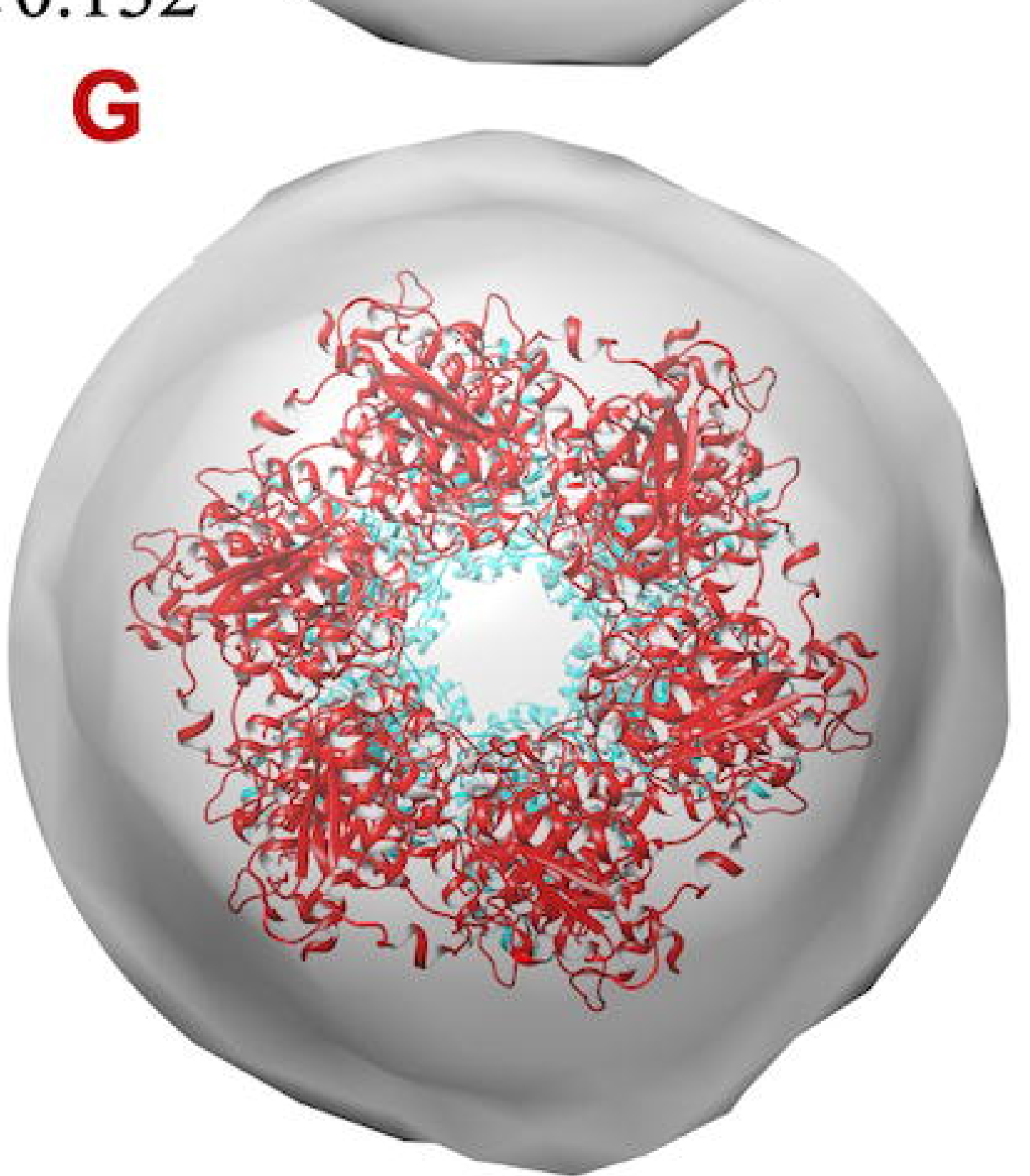

C Interatomic pairwise distribution function

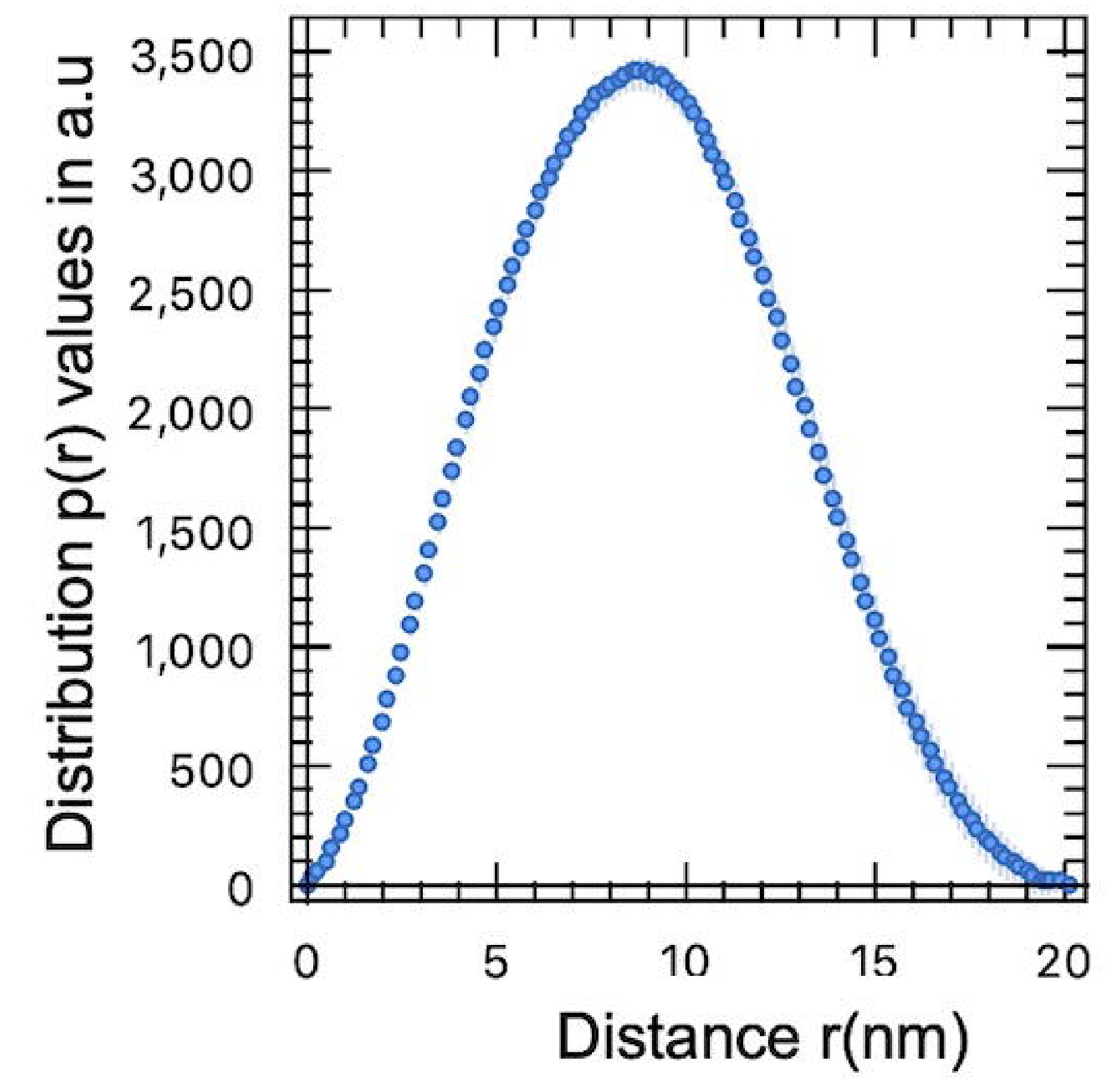

H

Model to fit experimental curve

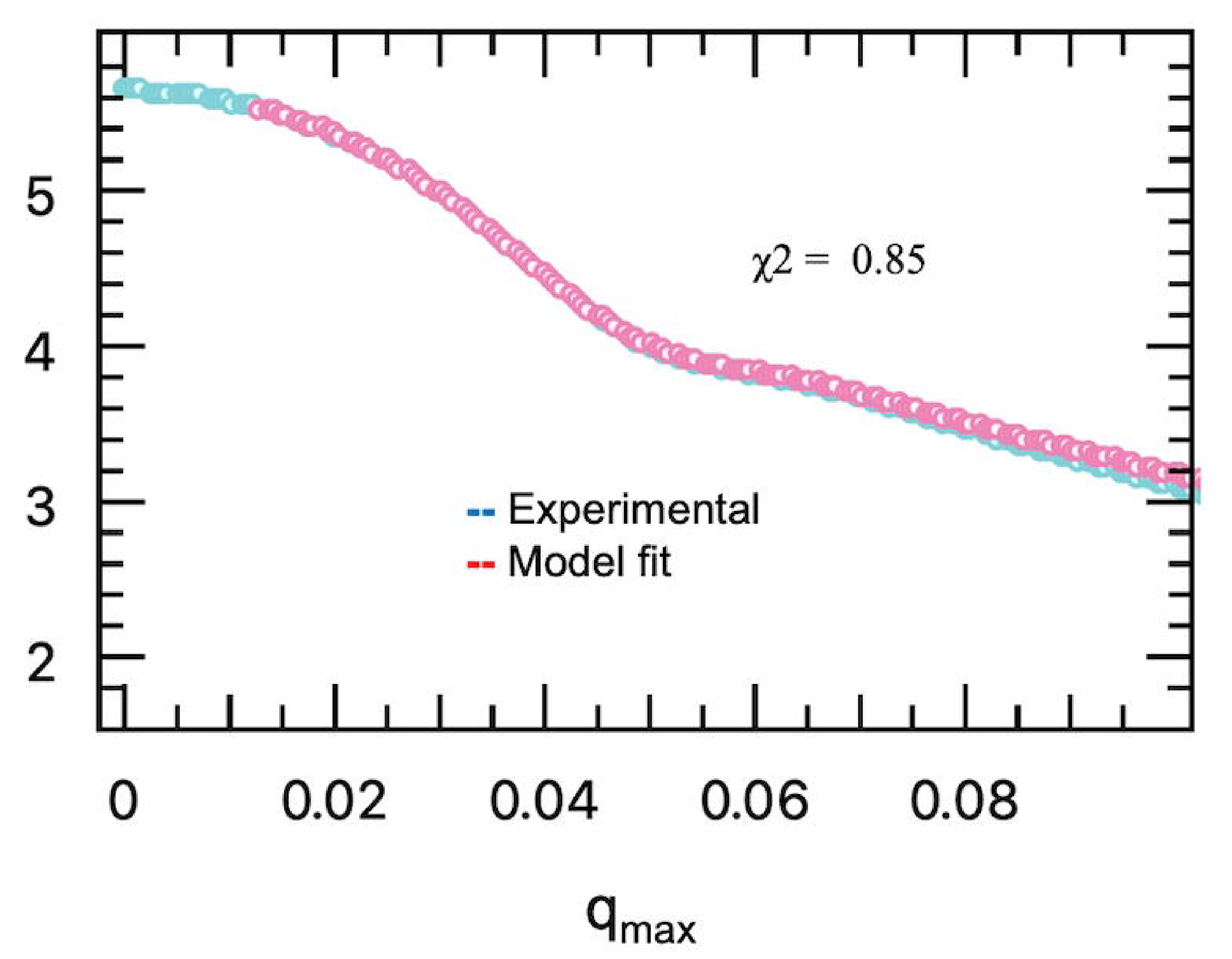


